
\title{
$\mathrm{UNLV} \mid \underset{\text { LIBRARIES }}{\mathrm{UNIVRSTT}}$
}

Chemistry and Biochemistry Faculty

Publications

Chemistry and Biochemistry

2012

\section{A New Method to Derive Electronegativity from Resonant Inelastic X-ray Scattering}

\author{
Stephane Carniato \\ University of Paris \\ Loic Journel \\ University of Paris \\ Renaud Guillemin \\ University of Paris, renaud.guillemin@upmc.fr \\ Maria Novella Piancastelli \\ Uppsala University, maria-novella.piancastelli@physics.uu.se \\ Wayne C. Stolte \\ University of Nevada, Las Vegas, wcstolte@lbl.gov \\ Follow this and additional works at: https://digitalscholarship.unlv.edu/chem_fac_articles \\ Cunextrage for additional authors \\ Part of the Biological and
}

\section{Repository Citation}

Carniato, S., Journel, L., Guillemin, R., Piancastelli, M. N., Stolte, W. C., Lindle, D. W., Simon, M. (2012). A New Method to Derive Electronegativity from Resonant Inelastic X-ray Scattering. Journal of Chemical Physics 1-13.

https://digitalscholarship.unlv.edu/chem_fac_articles/86

This Article is protected by copyright and/or related rights. It has been brought to you by Digital Scholarship@UNLV with permission from the rights-holder(s). You are free to use this Article in any way that is permitted by the copyright and related rights legislation that applies to your use. For other uses you need to obtain permission from the rights-holder(s) directly, unless additional rights are indicated by a Creative Commons license in the record and/ or on the work itself.

This Article has been accepted for inclusion in Chemistry and Biochemistry Faculty Publications by an authorized administrator of Digital Scholarship@UNLV. For more information, please contact digitalscholarship@unlv.edu. 
Authors

Stephane Carniato, Loic Journel, Renaud Guillemin, Maria Novella Piancastelli, Wayne C. Stolte, Dennis W. Lindle, and Marc Simon

This article is available at Digital Scholarship@UNLV: https://digitalscholarship.unlv.edu/chem_fac_articles/86 


\title{
A new method to derive electronegativity from resonant inelastic x-ray scattering
}

\author{
S. Carniato, ${ }^{1,2}$ L. Journel, ${ }^{1,2}$ R. Guillemin, ${ }^{1,2}$ M. N. Piancastelli, ${ }^{1,2, a)}$ W. C. Stolte, ${ }^{3,4,5}$ \\ D. W. Lindle, ${ }^{3,4}$ and M. Simon ${ }^{1,2}$ \\ ${ }^{1}$ UPMC Univ Paris 06, UMR7614, Laboratoire de Chimie Physique-Matière et Rayonnement, \\ 11 rue Pierre et Marie Curie, 75231 Paris Cedex 05, France \\ ${ }^{2}$ CNRS, LCPMR (UMR 7614), 11 rue Pierre et Marie Curie, 75231 Parix Cedex 05, France \\ ${ }^{3}$ Advanced Light Source, Lawrence Berkeley National Laboratory, Berkeley, California 94720, USA \\ ${ }^{4}$ Department of Chemistry, University of Nevada, Las Vegas, Nevada 89154-4009, USA \\ ${ }^{5}$ Harry Reid Center for Environmental Studies, University of Nevada, Las Vegas, Nevada, 89154-4003, USA
}

(Received 7 August 2012; accepted 18 September 2012; published online 10 October 2012)

\begin{abstract}
Electronegativity is a well-known property of atoms and substituent groups. Because there is no direct way to measure it, establishing a useful scale for electronegativity often entails correlating it to another chemical parameter; a wide variety of methods have been proposed over the past 80 years to do just that. This work reports a new approach that connects electronegativity to a spectroscopic parameter derived from resonant inelastic $\mathrm{x}$-ray scattering. The new method is demonstrated using a series of chlorine-containing compounds, focusing on the $\mathrm{Cl} 2 p^{-1} \mathrm{LUMO}^{1}$ electronic states reached after $\mathrm{Cl} 1 s \rightarrow$ LUMO core excitation and subsequent $K L$ radiative decay. Based on an electron-density analysis of the LUMOs, the relative weights of the $\mathrm{Cl} 2 p_{z}$ atomic orbital contributing to the $\mathrm{Cl} 2 p_{3 / 2}$ molecular spin-orbit components are shown to yield a linear electronegativity scale consistent with previous approaches. @ 2012 American Institute of Physics. [http://dx.doi.org/10.1063/1.4757065]
\end{abstract}

\section{INTRODUCTION}

Electronegativity is a fundamental concept closely coupled to chemical properties, such as bond energies, electron affinities, bond dipole moments, force constants, and inductive effects. In principle, direct correlations between electronstructure parameters and electronegativity should exist. Unfortunately, there is no direct experimental method to determine numerical values for electronegativity; they must either be calculated or derived from other chemical or physical properties. Several schemes have been proposed over the years, leading to a number of different electronegativity scales. ${ }^{1-15}$

Historically, three major approaches to electronegativity of atoms and chemical groups have emerged over the last century. In introducing the concept, Linus Pauling defined electronegativity as "the power of an atom in a molecule to attract electrons to itself,", $" 2$ i.e., the concept of electronegativity was clearly defined in terms of an atom within a molecule. In Pauling's thermochemical approach, $D(A-G)$, the energy of the $A-G$ bond in the polyatomic molecule $A-G$, is related to the bond energies $D(A-A)$ and $D(G-G)$ of the molecules $A-A$ and $G-G$, respectively, and to the difference between their electronegativities, $\Delta \chi=\chi_{A}-\chi_{G}$, by the relation

$$
D(A-G)=\sqrt{D(A-A) D(G-G)}+K(\Delta \chi)^{2} .
$$

Extension of this concept to groups of atoms gave rise to group electronegativity, ${ }^{5}$ in which a functional group can be treated as a pseudo-atom. Pauling's approach has been criticized because different factors (bond order, steric effects, etc.)

\footnotetext{
a) Permanent address: Department of Physics and Astronomy, Uppsala University, Box 516, 75120 Uppsala, Sweden.
}

likely contribute to the value of $\Delta \chi$. Furthermore, only the absolute value, $|\Delta \chi|$, can be derived directly from Eq. (1), leaving some ambiguity about its sign.

Later, in the context of density-functional theory, electronegativity (chemical potential) was identified by Iczkowski-Murgrave $^{16}\left(\right.$ Parr $\left.^{4}\right)$ as the negative (positive) of the partial derivative of the energy $\mathrm{E}$ of an atom or group of atoms with respect to the number of electrons $\mathrm{N}[-\mu=\chi$ $\left.=-(\partial \mathrm{E} / \partial \mathrm{N})_{v(R)}\right]$, determined within a constant external potential, $v(R)$. In this formalism, $\chi$ has units of energy, and it is considered to be a ground-state property of a free atom (or group of atoms), as opposed to the property of an atom within a molecule. An open question is how this latter approach might be related to measurable parameters. One possibility, in a Mulliken/Parr/Pearson framework, expresses the energy of an atom A with $Q$ electrons as

$E\left(Q_{A}\right)=E_{A}^{0}+\left(\frac{\partial E}{\partial Q_{A}}\right)_{Q_{A}^{0}} Q_{A}+1 / 2 Q_{A}^{2}\left(\frac{\partial^{2} E}{\partial Q_{A}^{2}}\right)_{Q_{A}^{0}}+\ldots$,

where $\frac{\partial E}{\partial Q_{A}}=\frac{I E+E A}{2}=\chi_{A}^{0}$ is the electronegativity (IE and EA are the vertical ionization energy and the electronic affinity, respectively) and $\frac{1}{2} \frac{\partial^{2} E}{\partial Q_{A}^{2}}=\frac{I E-E A}{2}=\eta_{A}$ is the hardness. Consequently, electrostatic energies in molecular systems are related not only to hardness and electronegativity but also are dependent on interatomic electrostatic energies within the molecule: ${ }^{17}$

$$
\begin{aligned}
E\left(Q_{1}, \ldots \ldots, Q_{N}\right)= & \sum_{A}\left(E_{A}^{0}+\chi_{A}^{0} Q_{A}+Q_{A}^{2} \eta_{A}\right) \\
& +\sum_{A<B} Q_{A} Q_{B} \mathrm{~J}_{A B},
\end{aligned}
$$


where $\mathrm{J}_{A B}$ is the Coulomb interaction between unit charges on centers $\mathrm{A}$ and $\mathrm{B}\left(\mathrm{J}_{A B}\right.$ depends on the distance between $\mathrm{A}$ and $B)$. Taking the derivative of $E$ with respect to $Q_{A}$ leads to an effective atomic-scale chemical potential of the form

$$
\chi_{A}=\partial E / \partial \mathrm{Q}_{A}=\chi_{A}^{0}+2 \eta_{A} \mathrm{Q}_{A}+\sum_{A \neq B} \mathrm{Q}_{B} \mathrm{~J}_{A B} .
$$

The Mulliken/Pearson electronegativity scale is based on IE and EA measurements in isolated atoms, radicals, or entire molecules. ${ }^{5}$ For A-G, where $A$ is an atom and $\mathrm{G}$ a group of atoms, determination of $\chi_{G}^{0}$ can thus suffer for radicals in which the geometry of the isolated species differs significantly from the geometry of the species bound in a molecule. This effect is not without consequences on the charge carried by atoms or radicals in molecular systems. Indeed, at equilibrium, where the atomic chemical potentials are equal ${ }^{8}$ and $\mathrm{Q}_{G}=-\mathrm{Q}_{A}$ :

$$
Q_{A}=\frac{\chi_{G}^{0}-\chi_{A}^{0}}{2\left(\eta_{G}+\eta_{A}-\mathrm{J}_{A G}\right)},
$$

where $\mathrm{J}_{A G}, \eta_{G}$, and $\chi_{G}^{0}$ are all dependent on the geometry of the group.

More recently, Allen 6 introduced an atomic "spectroscopic" electronegativity scale based on the average ionization energies of the valence electrons. For main-group (nontransition) elements,

$$
\chi=\frac{n_{s} \epsilon_{s}+n_{p} \epsilon_{p}}{n_{s}+n_{p}} .
$$

In Eq. (6), $\mathrm{n}_{s}$ and $\mathrm{n}_{p}$ are the numbers of $s$ and $p$ valence electrons, and $\epsilon_{s}$ and $\epsilon_{p}$ are the multiplet-averaged differences in total energy between the ground-state neutral atom and its monopositive ion resulting from the loss of a valence $s$ or $p$ electron. The quantities $\epsilon_{s}$ and $\epsilon_{p}$ can be extracted directly from spectroscopic data or approximated computationally, using appropriate orbital energies. While this approach has gained considerable acceptance for non-transition elements, it has been criticized because it ignores the effects of interpenetration of valence and shallow-core subshells, and because there is some ambiguity about the number of $d$ valence electrons to be used in calculating the configuration energies. While a new method has recently been proposed to mitigate these concerns, ${ }^{13}$ this third approach remains purely computational.

Generally, however, experiments typically focus on atoms or groups of atoms bound in a molecule, making it desirable to be able to correlate results from such measurements to electronegativity. For example, experimental observables measured by a variety of techniques, including bond-stretching frequencies obtained from infrared spectroscopy, ${ }^{18}$ NMR chemical shifts, ${ }^{19}$ isomer shifts in Mössbauer spectroscopy, ${ }^{20}$ and substituent constants due to field-induced effects $\left(\mathrm{F}^{21}\right.$ and $\left.\sigma_{F}^{22}\right)$ in benzene derivatives, have been shown to correlate with electronegativity. Numerous reports also have correlated $\chi$, and its related parameters (e.g., Hammett constants, which incorporate electronegativity and other factors such as polarizability and inductive effects), to core or valence binding energies measured via $\mathrm{x}$ ray or UV photoelectron spectroscopy (XPS or UPS). ${ }^{23-28}$ As an experimental method that interacts with electrons, photoelectron spectroscopy would seem to be a natural candidate to correlate to electronegativity because it probes directly the electronic structure of isolated molecules. In particular, coreshell results from XPS might be expected to correlate straightforwardly to electronegativity because of the well-known linear relationship between core binding energies and chemical shifts related to the local charge on the ionized atom.

Resonant inelastic x-ray scattering (RIXS) consists in analyzing in energy the radiative decay after resonant photoexcitation. An overview is presented of the theory of x-ray Raman scattering in Refs. 29 and 30, where a synopsis of relevant experimental techniques, and basic theoretical concepts and principles of X-ray Raman scattering are described. Because both RIXS and XPS are core-electron spectroscopies, properties (core binding energy, core-hole lifetime, ${ }^{31}$ electronegativity, etc.) derived from both techniques are sensitive to the net charge transfer in a molecule via relaxation after core excitation (or ionization ${ }^{32,33}$ ). RIXS deals with a neutral coreexcited state, as opposed to a core-ionized state in XPS. This is an important distinction because the degree to which the entire molecular medium is polarized will generally be different for a neutral state (RIXS) than a singly charged state (XPS).

The goal of this paper is to show that RIXS spectroscopy is an efficient way to probe charge sharing between an atom (in this case $\mathrm{Cl}$ ) and any ligand bound to it. The RIXS technique achieves this through promotion of a $\mathrm{Cl} 1 s$ electron to the lowest-unoccupied molecular orbital (LUMO) in an X-rayscattering process that reflects the character of the chemical bond, regardless of its polarizability. Using chlorinated compounds benefits from the fact that chlorine forms only single bonds, simplifying the interaction with its bonding partner and consequently the interpretation of the RIXS data. It is well established that the chemical environment of an atom in a molecule affects both its valence and core ionization energies. Subtler influences, such as spin-orbit coupling and molecular-field effects, also are routinely observed in molecular spectra. ${ }^{34}$ In chlorinated compounds, for example, the $\mathrm{Cl}-2 p$ spin-orbit coupling $(\approx 1.65 \mathrm{eV})$ is much larger than the molecular-field splitting, as is typical for core states. We have shown recently that radiative $K L$ decay in various chlorinecontaining compounds, specifically the $\mathrm{Cl} 1 s^{-1} \rightarrow 2 p^{-1}$ transition following resonant excitation of a $\mathrm{Cl} 1 s$ electron into the LUMO, exhibits complex structure and linear dichroism. ${ }^{35-40}$

A schematic representation of the present RIXS experiments is shown in Fig. 1. In $\mathrm{HCl}$, for instance, $K L$ emission spectra exhibit a characteristic spin-orbit doublet. ${ }^{41}$ However, the spin-orbit intensity ratio of the $2 p_{3 / 2}$ and $2 p_{1 / 2}$ components differs from the (atomic) statistical value of exactly 2 , and it varies as a function of the angle $\theta$ between the polarization of the incoming and scattered photons, ${ }^{36}$ with parallel polarization $\left(\theta=0^{\circ}\right)$ showing the largest deviation from 2 (see Fig. 1). These observations, particularly the linear dichroism, have been explained in terms of the electronic populations of the $\mathrm{Cl} 2 p_{z}$ and $2 p_{(x, y)}$ atomic orbitals contributing to the molecular spin-orbit doublet: essentially the linear combination of atomic orbitals in molecular orbitals picture applied to core states. The atomic $2 p_{z}$ and $2 p_{(x, y)}$ orbitals are inequivalent from a chemical point of view because the $2 p_{z}$ is 
oriented along the chemical bond, while $2 p_{x}$ and $2 p_{y}$ are perpendicular. Because the polarization of the scattered photons involved in the X-ray-scattering process, polarized-RIXS measurements provide a direct and sensitive determination of the relative weights of the atomic $2 p$ orbitals with different orientations relative to the bond axis.

Our experimental scheme involves creating a chlorine $2 p$ hole coupled to a spectator valence electron as final state. The same final state could also be reached by the direct excitation of a chlorine $2 p$ electron to the LUMO, and, thus, one might be able to get the same information from $\mathrm{x}$-ray absorption measurements (XAS), which would have the advantage of being a simpler experimental technique. However, RIXS measurements in the $\mathrm{Cl} K$-edge region $(\approx 2.8 \mathrm{keV})$ with long-pulse light sources produce high-energy-resolution decay spectra and can take advantage of ultrafast nuclear dynamics using the concept of effective duration time of the scattering process. ${ }^{29,30}$ We have previously shown $^{36,37,40,41}$ that the dynamical broadening $\left(\approx 1.20 \mathrm{eV}\right.$ in $\left.\mathrm{HCl}^{37}\right)$ observed in $\mathrm{x}$-ray absorption spectra is quenched on top of belowthreshold resonances, and the width of the x-ray-emission lines approaches the natural core-hole width $\left(\Gamma_{c} \approx 0.65 \mathrm{eV}\right)$. Line narrowing is more apparent for transitions between core shells, such as $\mathrm{Cl} 2 p$ and $1 s$, because these core-excited states have nearly parallel potential-energy surfaces. ${ }^{41}$ Studies of such transitions have revealed that $\mathrm{Cl} 2 p_{z}$ substructures in spin-orbit $K L$ lines of fundamental interest in the present study, ${ }^{35-40}$ have very low cross-section and can not be relevant (see Fig. 1 and Ref. 37 for details) in x-ray absorption spectroscopy. depends directly on the symmetry of the initial and final states

In this work, we report spin-orbit-state populations obtained both theoretically and experimentally for a series of chlorine-containing molecules. Experimental values were collected for $\mathrm{HCl}, \mathrm{CF}_{3} \mathrm{Cl}^{36,37} \mathrm{Cl}_{2}$, and $\mathrm{CH}_{3} \mathrm{Cl}^{39}$ using polarized-RIXS measurements. For these systems, theoretical ab-initio calculations and Eq. (25) in Refs. 36 and 37 explained the physics and reproduced the experimental variation of the $2 p_{3 / 2}$ and $2 p_{1 / 2}$ intensity ratios as a function of polarization.

New theoretical results are presented here for a much wider variety of Cl-R molecules, with $\mathrm{R}=\mathrm{K}, \mathrm{Na}, \mathrm{Li}, \mathrm{SiH}_{3}$, $\mathrm{SiCl}_{3}, \mathrm{I}, \mathrm{t}-\mathrm{C}_{4} \mathrm{H}_{9}, \mathrm{C}_{2} \mathrm{H}_{5}, \mathrm{i}-\mathrm{C}_{3} \mathrm{H}_{7}, \mathrm{C}\left(\mathrm{NH}_{2}\right)_{3}, \mathrm{H}, \mathrm{CH}_{3}, \mathrm{Br}, \mathrm{NH}_{2}$, $\mathrm{SF}_{5}, \mathrm{Cl}, \mathrm{NF}_{2}, \mathrm{OCH}_{3}, \mathrm{SiF}_{3}, \mathrm{CF}_{3}, \mathrm{OH}, \mathrm{C}\left(\mathrm{NO}_{2}\right)_{3}$, and F. For all of these species, relative weights of the $\mathrm{Cl} 2 p_{z}$ atomic orbital contributing to the $2 p_{3 / 2}$ molecular spin-orbit state were compared to electronegativities of the substituent atoms or groups bonded to chlorine, and a linear relationship was found between tabulated Pauling/Datta values of electronegativity and the $2 p_{z}$ populations. In addition, the energy splitting of the constituent $2 p_{z}$ and $2 p_{x, y}$ atomic orbitals is sensitive to the nature of the ligand as well as the Cl-R bond distance, thus influencing the strength of the mixing of triplet and singlet states by the spin-orbit interaction. These findings are explained in terms of the localization of the electron excited to the LUMO in each species.

While electronegativity scales are numerous and the literature extensive, the goal of the present paper is to discuss to which extent links between different scales can be made. In other words, is there a direct connection between the electronegativity of the substituent groups and the observed $2 p_{z}$ population relative to the core-excited chlorine atom?
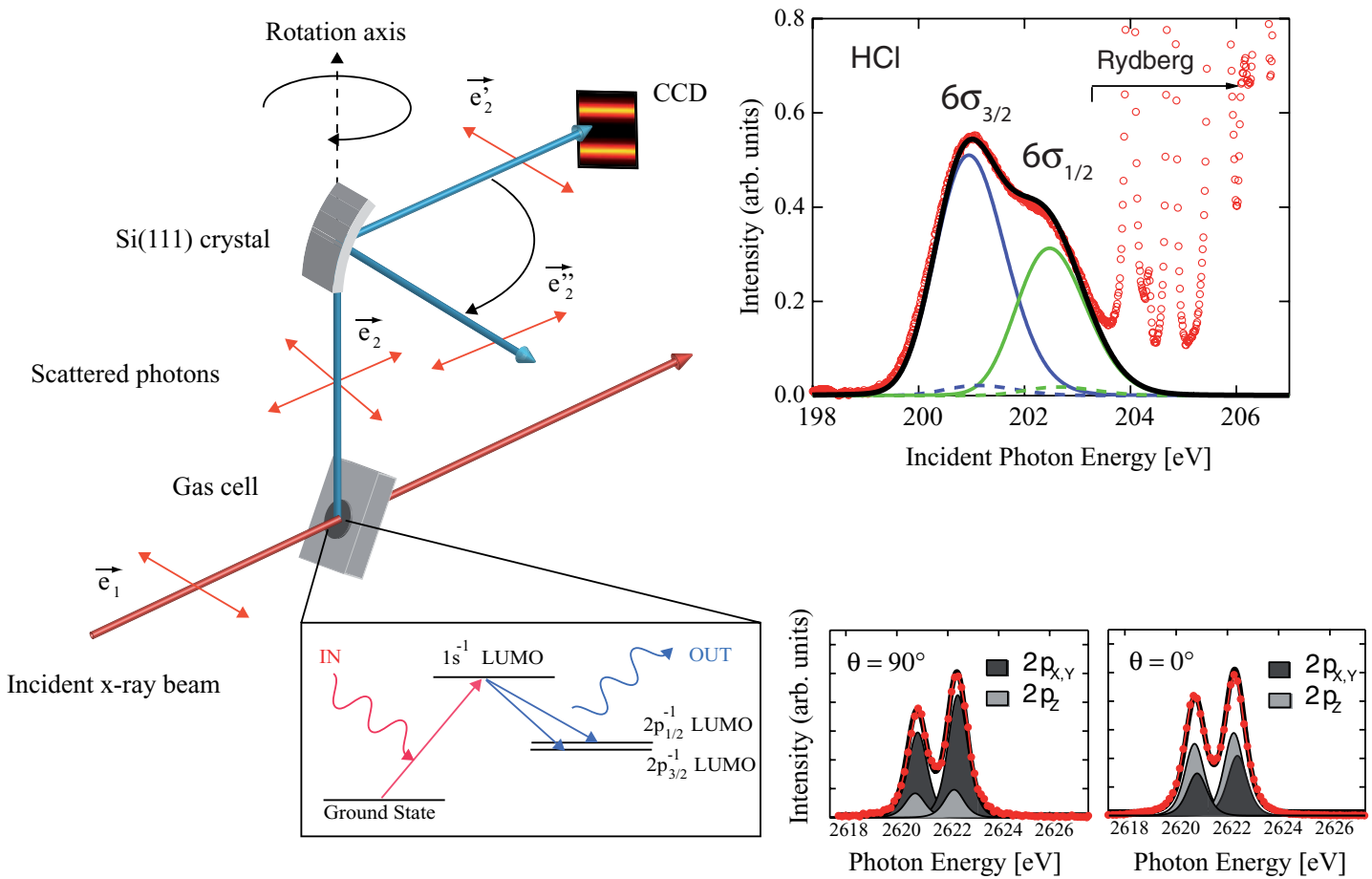

FIG. 1. Left panel: Schematic of the polarized-x-ray emission spectrometer used for RIXS measurements. Inset: Simplified representation of the RIXS process studied. (See text for details.) Right panel (upper): Experimental and calculated X-ray absortpion spectra of $\mathrm{HCl}^{37}$ In solid lines (dashed line) are indicated the $2 p_{3 / 2}$ and $2 p_{1 / 2}$ spin-orbit states where the $2 p$ hole is in the $\mathrm{Cl} 2 p_{x y}\left(\mathrm{Cl} 2 p_{z}\right)$ atomic orbital. Right panel (lower): Theoretical $K L$ RIXS spectra obtained at $\theta$ $=0^{\circ}$ (left) and $\theta=90^{\circ}$ (right) at the top of the resonance, compared to experiment. $\mathrm{Cl} 2 p_{z}$ components of interest are in grey colour. 


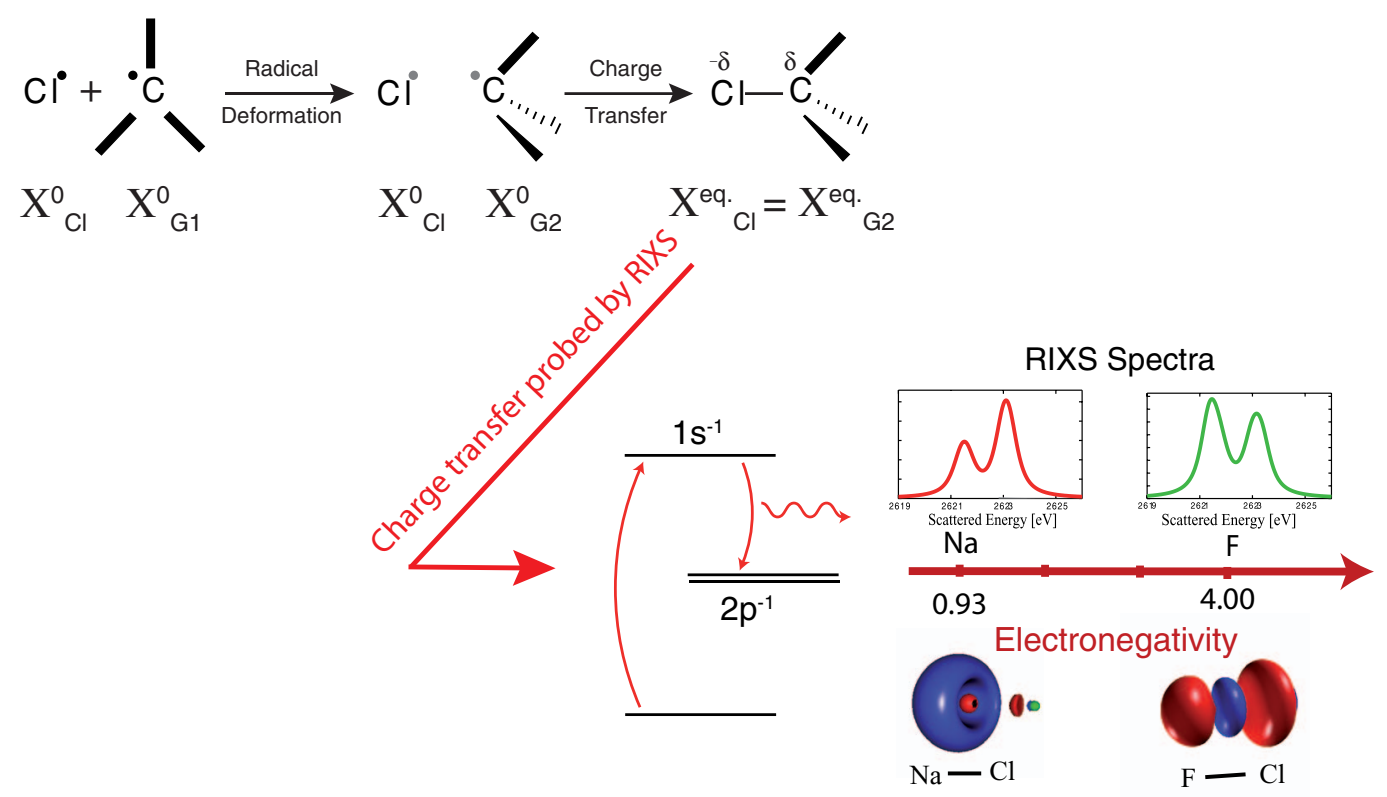

FIG. 2. Pictorial description of the relationship between absolute electronegativities in free atoms/radicals and the electronegativity scale derived in the present work. $\mathrm{G}_{1}$ and $\mathrm{G}_{2}$ refer to different geometries of the same radical.

\section{RESULTS AND DISCUSSION}

\section{A. RIXS-derived electronegativities: Probing electron-density sharing}

Figure 2 shows a pictorial representation of the charge transfer probed by RIXS and the general scheme of how the experimental results relate to electronegativity.

Because the RIXS final state includes two unpaired electrons (one core, one valence), molecular $\mathrm{Cl} 2 p$ spin-orbit states are a mixture of nonrelativistic atomic $2 p_{x, y, z}$ singlet and triplet states, with the strength of the singlet-triplet mixing being strongly related to the spin-orbit coupling parameter for the $\mathrm{Cl} 2 p$ subshell and the singlet-triplet exchange energy, $\Delta \mathrm{ST}$ (see Sec. III B). The splitting $\Delta \mathrm{ST}$ can be formulated as twice the integral exchange energy (due to exchange, the triplet state is lower than the corresponding singlet state):

$\Delta \mathrm{ST}=2 \iint \phi_{2 p}^{*}(1) \Psi_{L U M O}^{*}(2) \frac{1}{r_{12}} \phi_{2 p}(2) \Psi_{L U M O}(1) d \mathbf{r}_{1} d \mathbf{r}_{2}$.

Considering a two-level model where the antibonding LUMO is constructed as a linear combination of chlorine and ligand atomic orbitals, $\Psi_{L U M O}=c_{A} \phi_{A}+c_{B} \phi_{B}$, centered on atoms $\mathrm{A}(\mathrm{Cl})$ and $\mathrm{B}(\mathrm{R})$, respectively, the exchange integral can be rewritten as

$$
\begin{aligned}
& \iint \phi_{2 p_{A}}^{*}(1) \Psi_{L U M O}^{*}(2) \frac{1}{r_{12}} \phi_{2 p_{A}}(2) \Psi_{L U M O}(1) d \mathbf{r}_{1} d \mathbf{r}_{2} \\
& =c_{A}^{2} I_{2 p A}+c_{B}^{2} I_{2 p B}+2 c_{A} c_{B} I_{2 p A B},
\end{aligned}
$$

where

$$
I_{2 p A(B)}=\iint \phi_{2 p_{A}}^{*}(1) \phi_{A(B)}^{*}(2) \frac{1}{r_{12}} \phi_{2 p_{A}}(2) \phi_{A(B)}(1) d \mathbf{r}_{1} d \mathbf{r}_{2}
$$

and

$$
I_{2 p A B}=\iint \phi_{2 p_{A}}^{*}(1) \phi_{A}^{*}(2) \frac{1}{r_{12}} \phi_{2 p_{A}}(2) \phi_{B}(1) d \mathbf{r}_{1} d \mathbf{r}_{2},
$$

where $I_{2 p A(B)}$ and $I_{2 p A B}$ are the one-site and two-site atomic exchange integrals, respectively.

In principle, $I_{2 p B}$ is a small quantity and $\Delta S T$ exchange integral mainly depends on the localized or diffuse character of $I_{2 p A}, I_{2 p A B}$ as well as the relative magnitude of $\mathrm{c}_{A}$, and $\mathrm{c}_{B}$ coefficients.

As already depicted, RIXS measurable quantity is a spectroscopic spin-orbit subcomponent $\left(2 p_{z}\right.$ weigths in $\mathrm{KL}$ in chlorinated compounds). Because the final state in the RIXS process bears a core hole in the $\mathrm{Cl} 2 p$ subshell as well as in XPS core-ionized process, factors beyond electronegativity and chemical hardness (or group polarizability) must be considered, a priori, when interpreting RIXS phenomenon. These factors include charge transfer within the molecule due to electronic relaxation/correlation induced of valence electrons and re-polarization of excited electron in the LUMO in the presence of the core hole.

The amount of charge transfer that occurs through repolarization of the valence electrons on the spectator chlorine can be significant (see Mulliken charges $\mathrm{Q}_{C l}$ in the ground state and $\mathrm{Q}_{C l}^{2 p z}$ in the $2 p_{z}^{-1} L U M O^{1}$ core-excited state reported in Table I). According to the core-equivalent model, ${ }^{42}$ RIXS and XAS scales can be viewed as related to the properties of a neutral Ar-R species. In general, the spectator electron tends to be polarized away from the core hole and the valence electrons towards the core hole. This phenomenon has been already discussed in the literature. ${ }^{43}$ As shown in Fig. 3 and Table I, and although one has to take care from Mulliken charges partitioning, our analysis over a large set of radicals shows that the $2 p_{z}$ weigths are well connected with the charge distribution between the atomic chlorine 
TABLE I. Calculated (this work) and experimental ${ }^{36,37,39,40}$ populations (in $\%$ ) of the singlet $\mathrm{Cl} 2 p_{z}^{-1} \mathrm{LUMO}^{1}$ component in the $\mathrm{Cl}_{2} 2 p_{3 / 2}^{-1} \mathrm{LUMO}^{1}$ spin-orbit state. Hartree-Fock singlet-triplet energy differences $(\Delta \mathrm{ST})$, molecular-field splittings (MF) $\left(\mathrm{E} 2 \mathrm{p}_{x y}-\mathrm{E} 2 \mathrm{p}_{z}\right)$ in the ground state (GS) and Mulliken Cl elect. pop. $\mathrm{Pop}(\mathrm{Cl})^{L U M O}$ on the LUMO in the $\mathrm{Cl} 2 p_{z}^{-1} \mathrm{LUMO}^{1}$ final state. Mulliken $\mathrm{Q}_{C l}$ and $\mathrm{Q}_{C l}^{2 p z}$ on chlorine in $\mathrm{GS}$ and $2 p_{z}^{-1} \mathrm{LUMO}^{1}$ core-excited states.

\begin{tabular}{|c|c|c|c|c|c|c|c|c|c|c|c|}
\hline \multirow[b]{2}{*}{ Group } & \multicolumn{2}{|c|}{$2 p_{z}$ pop. $(\%)$} & \multirow{2}{*}{$\begin{array}{l}\Delta \mathrm{ST} \\
(\mathrm{eV})\end{array}$} & \multirow{2}{*}{$\begin{array}{l}\text { MF } \\
(\mathrm{eV})\end{array}$} & \multirow[b]{2}{*}{$\operatorname{Pop}(\mathrm{Cl})^{L U M O}$} & \multirow[b]{2}{*}{$\mathrm{Q}_{C l}$} & \multirow[b]{2}{*}{$\mathrm{Q}_{C l}^{2 p z}$} & \multicolumn{4}{|c|}{ Electronegativity $\chi$} \\
\hline & Theory & Exp. & & & & & & $\mathrm{A}^{\mathrm{c}}$ & $\mathrm{XPS}^{\mathrm{a}}$ & $B^{d}$ & This work \\
\hline $\mathrm{K}$ & 64.7 & & 0.01 & 0.005 & -0.09 & -0.89 & 0.090 & 2.42 & & 0.82 & 0.92 \\
\hline $\mathrm{Na}$ & 64.6 & & 0.02 & 0.005 & -0.03 & -0.84 & -0.087 & 2.85 & & 0.93 & 0.93 \\
\hline $\mathrm{Li}$ & 64.5 & & 0.01 & 0.005 & -0.04 & -0.66 & 0.062 & 3.01 & & 0.98 & 0.95 \\
\hline $\mathrm{SiH}_{3}$ & 57.9 & & 0.21 & 0.027 & $\approx 0.0$ & -0.39 & 0.400 & 4.78 & 2.38 & 1.76 & 1.70 \\
\hline $\mathrm{SiCl}_{3}$ & 54.6 & & 0.32 & 0.030 & 0.02 & -0.25 & 0.306 & & & & 2.09 \\
\hline I & 53.3 & & 0.25 & 0.049 & 0.06 & -0.41 & -0.003 & 6.76 & 2.62 & 2.66 & 2.24 \\
\hline $\mathrm{t}-\mathrm{C}_{4} \mathrm{H}_{9}$ & 53.2 & & 0.34 & 0.030 & 0.23 & -0.45 & -0.101 & 3.31 & 1.93 & 2.65 & 2.25 \\
\hline $\mathrm{C}\left(\mathrm{NH}_{2}\right)_{3}$ & 52.9 & & 0.35 & 0.030 & 0.24 & -0.22 & 0.063 & & & & 2.28 \\
\hline $\mathrm{i}-\mathrm{C}_{3} \mathrm{H}_{7}$ & 52.4 & & 0.36 & 0.032 & 0.29 & -0.35 & -0.075 & 3.55 & 2.06 & 2.54 & 2.34 \\
\hline $\mathrm{Br}$ & 52.2 & & 0.35 & 0.050 & 0.12 & -0.12 & 0.006 & 7.60 & & 2.96 & 2.37 \\
\hline $\mathrm{H}$ & 52.0 & $\mathbf{5 0 . 0 ^ { b }}$ & 0.35 & 0.043 & 0.38 & -0.10 & 0.105 & 7.17 & 3.00 & 2.20 & $2.39 / 2 . \mathbf{6 2}^{\mathrm{b}}$ \\
\hline $\mathrm{C}_{2} \mathrm{H}_{5}$ & 51.9 & & 0.38 & 0.033 & 0.29 & -0.36 & -0.040 & 4.00 & 2.22 & 2.59 & 2.40 \\
\hline $\mathrm{CH}_{3}$ & 51.0 & $\mathbf{5 2 .} .0^{\mathrm{b}}$ & 0.39 & 0.035 & 0.30 & -0.33 & 0.005 & 4.96 & 2.40 & 2.68 & $2.50 / \mathbf{2} . \mathbf{. 3 9}^{\mathrm{b}}$ \\
\hline $\mathrm{SiF}_{3}$ & 50.3 & & 0.34 & 0.032 & $\ldots$ & -0.07 & 0.572 & & & & 2.59 \\
\hline $\mathrm{CCl}_{3}$ & 48.8 & & 0.45 & 0.062 & 0.33 & -0.02 & 0.216 & & 2.81 & & 2.76 \\
\hline $\mathrm{NF}_{2}$ & 48.8 & & 0.43 & 0.054 & 0.25 & 0.17 & 0.245 & & & 3.18 & 2.76 \\
\hline $\mathrm{Cl}$ & 48.7 & $46.0^{\mathrm{b}}$ & 0.41 & 0.005 & 0.21 & 0.00 & 0.167 & 8.31 & 3.22 & 3.16 & $2.77 / 3.09^{b}$ \\
\hline $\mathrm{NH}_{2}$ & 48.5 & & 0.43 & 0.052 & 0.30 & -0.03 & 0.12 & 6.07 & & 3.10 & 2.79 \\
\hline $\mathrm{SF}_{5}$ & 47.3 & & 0.37 & 0.043 & 0.25 & -0.16 & 0.147 & & 3.02 & & 2.93 \\
\hline $\mathrm{OCH}_{3}$ & 46.9 & & 0.47 & 0.057 & 0.32 & 0.24 & 0.21 & & 3.40 & & 2.98 \\
\hline $\mathrm{CF}_{3}$ & 46.5 & $46.5^{\mathrm{b}}$ & 0.48 & 0.043 & 0.34 & -0.04 & 0.188 & & 3.23 & 2.58 & $3.02 / \mathbf{3 . 0 2}^{\mathrm{b}}$ \\
\hline $\mathrm{OH}$ & 44.5 & & 0.50 & 0.060 & 0.31 & 0.09 & 0.320 & 7.50 & & 3.46 & 3.26 \\
\hline $\mathrm{C}\left(\mathrm{NO}_{2}\right)_{3}$ & 41.4 & & 0.55 & 0.052 & 0.38 & 0.09 & 0.32 & & & & 3.61 \\
\hline $\mathrm{F}$ & 38.1 & & 0.64 & 0.076 & 0.45 & 0.61 & 0.422 & 10.41 & 3.95 & 3.98 & 4.00 \\
\hline
\end{tabular}

${ }^{a}$ Values from $\mathrm{Cl} 2 p_{3 / 2} \mathrm{BE}$ as in Ref. 33.

${ }^{\mathrm{b}}$ Experimental values from polarized-RIXS measurements.

${ }^{\mathrm{c}} \mathrm{A}$ from Ref. 5 (in eV).

${ }^{\mathrm{d}}$ B from Ref. 11

and the radical in the neutral ground state. Furthermore, as clearly depicted by Eq. (5), i.e., by changing $\chi_{C l}^{0}(8.30 \mathrm{eV}) /$ $\eta_{C l}(4.68 \mathrm{eV})$ with $\chi_{A r}^{0}(7.698 \mathrm{eV}) / \eta_{A r}(8.061 \mathrm{eV})$, as far as the effect of changing $\mathrm{Cl}$ by $\mathrm{Ar}$ can modify the magnitude (and even the sign) of the charge on chlorine, different $\mathrm{Pop}(\mathrm{Cl})^{L U M O} \rightarrow \Delta(S T) \rightarrow 2 p_{z}$ still persist.

Thus, RIXS spectroscopy reflects the character of the chemical bond by probing the charge sharing between excited (argon-like) atomic chlorine and its bonding partner, R, via the promotion of an electron to the LUMO, regardless of the polarizability of the molecule.

Figure 4 depicts more precisely the relationship between LUMO electron densities and the derived electronegativity scale. In the following discussion, we consider the three cases shown in Fig. 4, for which the difference in electronegativity $(\Delta \chi)$ between $\mathrm{Cl}$ and the ligand $\mathrm{R}$ is large and positive $(\mathrm{R}=\mathrm{Na})$, large and negative $(\mathrm{R}=\mathrm{F})$, and small $\left(\mathrm{R}=\mathrm{CF}_{3}\right)$.

When chlorine is more electronegative $\left(\chi_{C l}>\chi_{R}\right.$, e.g., in alkali systems such as $\mathrm{LiCl}, \mathrm{NaCl}$, and $\mathrm{KCl}$ ), $\mathrm{R}$ acts as an electron-donating group in the ground state. As shown in Fig. 4 for $\mathrm{NaCl}$, the LUMO is localized mainly on $\mathrm{Na}$, and the probability of finding the excited electron near the chlorine atom is small (see Table I, the LUMO Mulliken population on the chlorine atom, $\left.\operatorname{Pop}(\mathrm{Cl})^{L U M O} \approx 0.0\right)$, resulting in nearly degenerate singlet and triplet states and a small singlet-triplet exchange energy $(\Delta \mathrm{ST} \sim 0.02 \mathrm{eV})$.
Alternatively, if chlorine is less electronegative than $\mathrm{R}$ $\left(\chi_{C l}<\chi_{R}\right), \Delta \mathrm{ST}$ is much larger and is correlated to the degree of localization of the LUMO on the chlorine atom. For

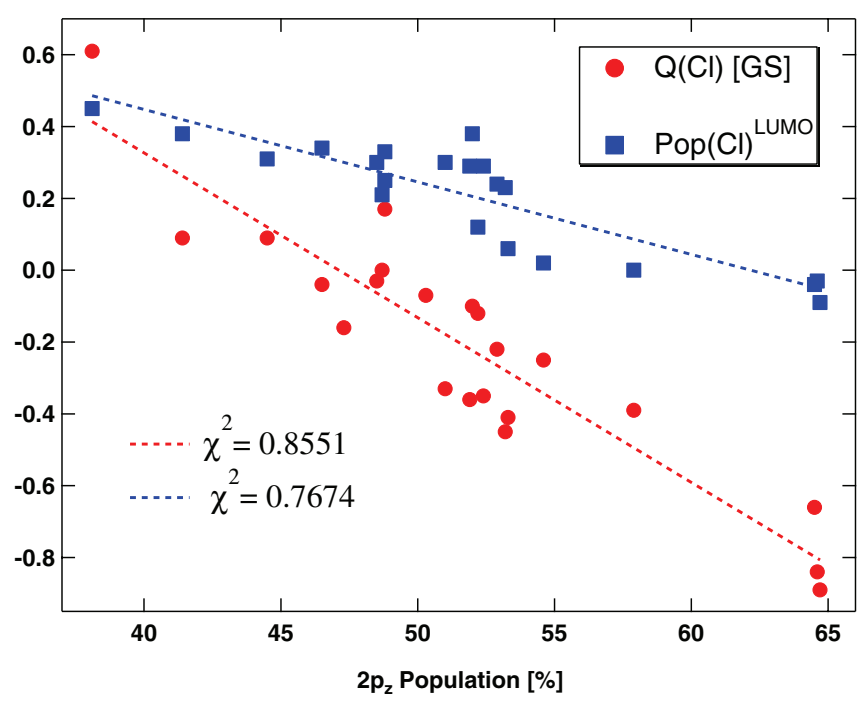

FIG. 3. Calculated Hartree-Fock Mulliken charge, $\mathrm{Q}_{\mathrm{Cl}}$ (in red), in the ground state and electronic population $\left(\operatorname{Pop}(\mathrm{Cl})^{L U M O}\right)$ on chlorine in the $2 \mathrm{p}_{z}^{-1} L U M O^{1}$ core-excited state (in blue) vs $\% 2 \mathrm{p}_{z}^{-1} L U M O^{1}$ spin-orbit population. 
Final state $2 \mathrm{p}^{-1} \mathrm{LUMO}^{+1} \quad$ LUMO $\quad$ RIXS Spectra
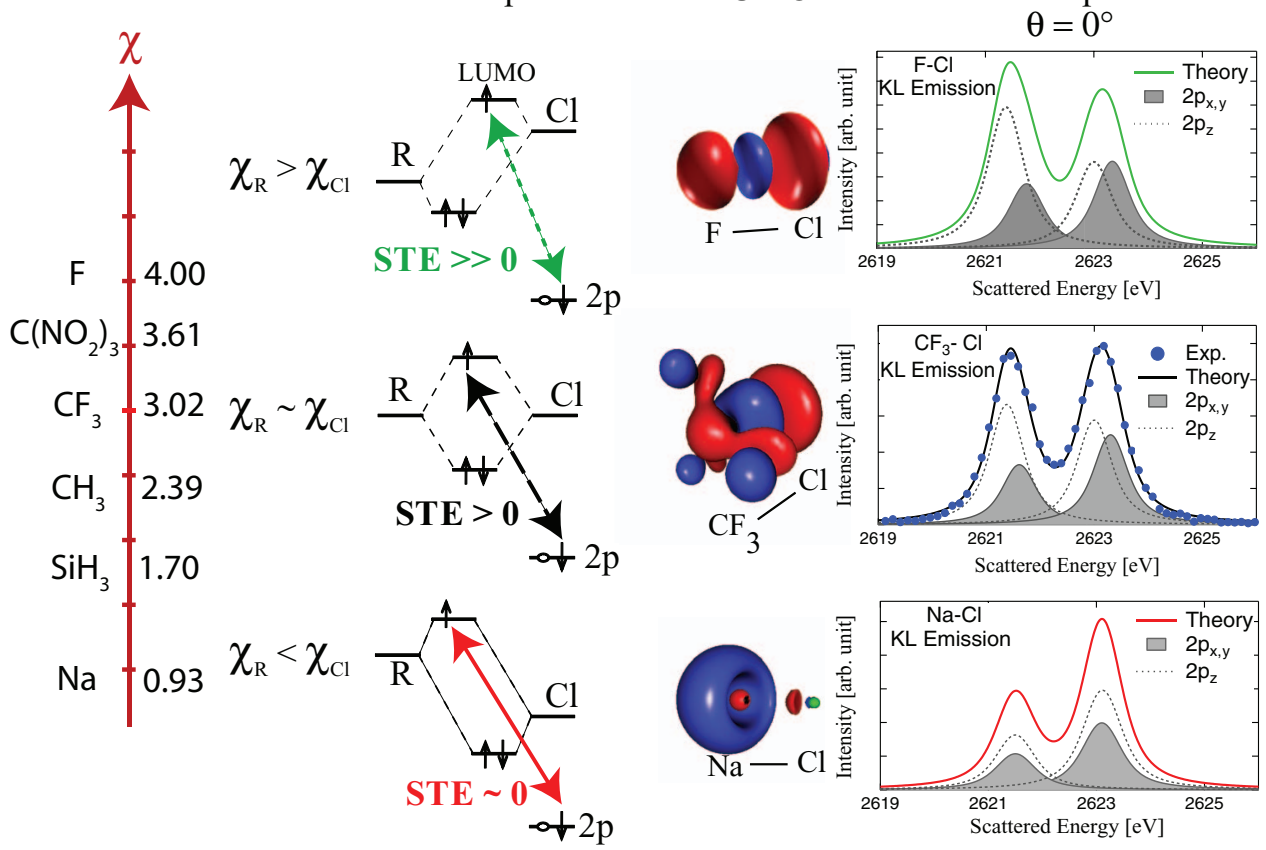

FIG. 4. Pictorial description of the relationship between various chemical properties: (i) electronegativity scale derived in the present work; (ii) singlettriplet exchange (STE) interactions and LUMO electron-density surfaces $\left(0.04 \mathrm{e} / \AA^{3}\right)$ for $2 p^{-1} \mathrm{LUMO}^{1}$ core-excited states of three different $\mathrm{Cl}$-R molecules; (iii) calculated polarized-RIXS $K L$ spectra $(\theta=0)$ for $\mathrm{NaCl}, \mathrm{CF}_{3} \mathrm{Cl}$, and $\mathrm{Cl}-\mathrm{F}$ from Eq. (27). Comparison is made with the experimental spectrum for $\mathrm{CF}_{3} \mathrm{Cl}$. Incoming photon bandwidth, $\gamma_{p}=0.2 \mathrm{eV}$, and spectrometer resolution, $\gamma_{s}=0.25 \mathrm{eV}$, as half width half maximum (HWHM) have been used for the calculations.

core excitation in $\mathrm{Cl}-\mathrm{F}$, for example, the excited electron in the LUMO is mainly on chlorine $\left(\mathrm{Pop}(\mathrm{Cl})^{L U M O}=0.45\right)$. As seen in the theoretical RIXS spectrum of Cl-F (Fig. 4), each spin-orbit peak splits into two relativistic components. At the equilibrium bond length $\left(d_{C l-F}=1.638 \AA\right)$, the calculated energy splitting between the two states in $2 p_{3 / 2}$ is $0.34 \mathrm{eV}$, and it is slightly larger $(0.38 \mathrm{eV})$ for $2 p_{1 / 2}$. In this molecule, the singlet-triplet splitting $(\Delta \mathrm{ST} \sim 640 \mathrm{meV})$ far exceeds the $2 p$ molecular-field splitting $(76 \mathrm{meV})$ in the ground state. As a result, an inversion of the $2 p_{3 / 2} / 2 p_{1 / 2}$ spin-orbit ratio, to a value less than unity, is predicted for $\mathrm{Cl} K L$ spectra of Cl-F.

Between these two extremes, for intermediate values of $\Delta \chi$, when a $\mathrm{Cl} 1 s$ core electron is promoted to the LUMO, the degree of delocalization of the electron density along the $\mathrm{R}-\mathrm{Cl}$ bond (ex: $\mathrm{Pop}(\mathrm{Cl})^{L U M O}=0.30$ for $\mathrm{Cl}$ in $\mathrm{CH}_{3} \mathrm{Cl}$ ) is directly related to $\triangle \mathrm{ST}$. In other words, the electron promoted to the antibonding LUMO acts as a convenient measure of charge transfer between the two bound species, and thus of their difference in electronegativity.

Experimental and theoretical $K L$ RIXS spectra, with an incident X-ray energy tuned to the $\mathrm{Cl} 1 s \rightarrow$ LUMO resonance in $\mathrm{CF}_{3} \mathrm{Cl}(2823.5 \mathrm{eV})$, are shown in Fig. 5 for both parallel $(\|)$ and perpendicular $(\perp)$ polarization (see Fig. 1). Also shown is the dependence of the $\mathrm{Cl} 2 p$ spin-orbit ratio as a function of the angle $\theta$ between the polarization vectors of the incident and scattered photons. The measured ratios are $R_{\perp}=1.30$ \pm 0.05 and $R_{\|}=1.07 \pm 0.05$.

Recently, we have shown $^{36,37,39,40}$ that it is possible to extract directly from this measured linear dichroism the singlet populations $\left(W_{2 p_{3 / 2}, S}^{z}\right.$ and $W_{2 p_{3 / 2}, S}^{(x, y)}$, respectively) of the $\mathrm{Cl} 2 p_{z}^{-1} \mathrm{LUMO}^{1}$ and $\mathrm{Cl} 2 p_{x, y}^{-1} \mathrm{LUMO}^{1}$ nonrelativistic configurations contributing to each of the molecular spinorbit components. Details of this procedure are presented in Secs. III B and III D.

We report in Table I relative weights, $W_{2 p_{3 / 2}, S}^{z}$, of the nonrelativistic $\mathrm{Cl} 2 p_{z}$ atomic orbital (in \%) contributing to the $2 p_{3 / 2}^{-1} \mathrm{LUMO}^{1}$ spin-orbit final states of the RIXS process, calculated at the equilibrium bond distances for a variety of chlorine-containing molecules. Because of alignment with respect to the Cl-R bond, the contribution of the $2 p_{z}$ atomic

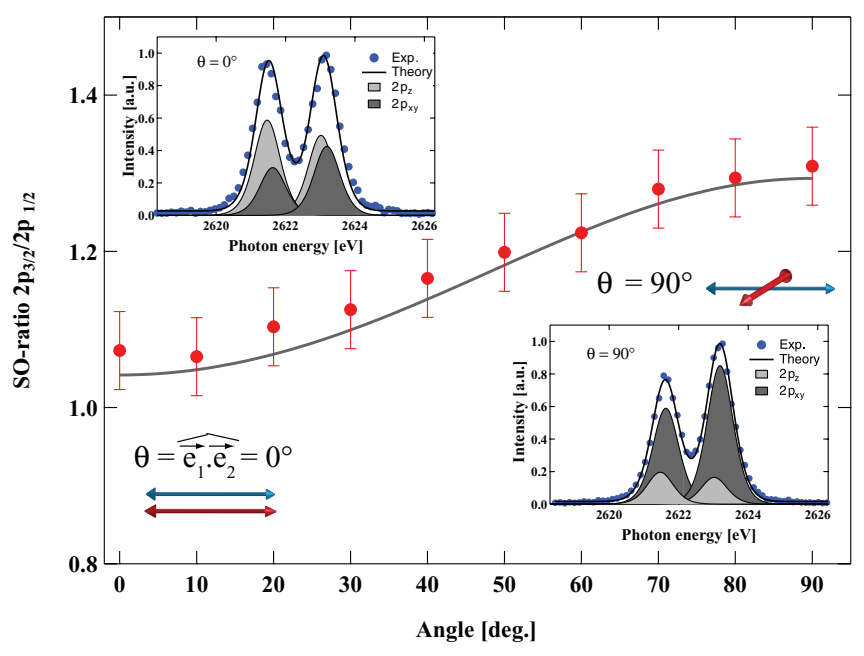

FIG. 5. Experimental $2 p_{3 / 2} / 2 p_{1 / 2}$ spin-orbit intensity ratios (circles) from $\mathrm{CF}_{3} \mathrm{Cl} K L$ RIXS spectra as a function of the angle $\theta$ between the polarization vectors of the incident and scattered photons, compared to calculated values (solid curve). Insets: Theoretical $K L$ RIXS spectra obtained at $\theta=0^{\circ}$ (left) and $\theta=90^{\circ}$ (right), compared to experiment. 


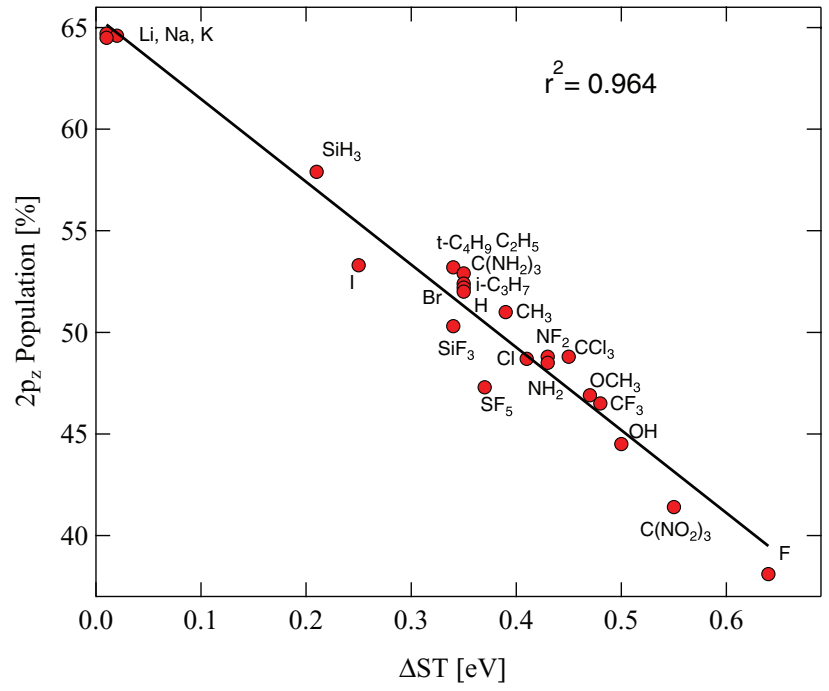

FIG. 6. Atomic $\mathrm{Cl} 2 p_{z}$ populations as a function of the singlet-triplet exchange energy $\Delta \mathrm{ST}$.

component to the $2 p_{3 / 2}$ molecular state is more sensitive to the nature of the group R (varying from $66 \%$ to $38 \%$ for the systems investigated) than the $2 p_{(x, y)}$ components (not shown, but ranging only from $57 \%$ to $65 \%$ ). For $\mathrm{HCl}, \mathrm{CF}_{3} \mathrm{Cl}^{36,37,40}$ and $\mathrm{CH}_{3} \mathrm{Cl},{ }^{39}$ the singlet-triplet splitting, $\Delta \mathrm{ST}$, also was shown to be correlated strongly to interatomic distance; similarly to the $2 p_{z}$ population, $\Delta \mathrm{ST}$ depends on the nature of the chemical bond, but with the chlorine character of the LUMO being anti-correlated to $\Delta \mathrm{ST}$. Indeed, the theoretical and experimental data collected in Table I show an inverse linear relationship between the $2 p_{z}$ singlet populations and $\Delta \mathrm{ST}$, as illustrated in Fig. 6.

Thus, a key result of this work is that $\mathrm{Cl} 2 p_{z}$ populations fit reasonably well to a linear expression with respect to the singlet-triplet exchange energy $\Delta \mathrm{ST}$ for a wide variety of chemical species bound to a chlorine atom. We note that theoretical values for these populations agree quite well with those determined from experiment. As a result, for systems in which experimental results are unavailable, theory is expected to provide acceptable values. Finally, both of these complementary parameters depend on the electronegativity difference $\Delta \chi$ between the chlorine atom and the ligand $\mathrm{R}$, a notion discussed in more detail below. From this result, one can develop an alternate electronegativity scale based on $2 p_{z}$ populations.

We plot in Fig. 7 the present electronegativity values versus Pauling's for a number of species. For the sake of illustration, we have normalized to Pauling's electronegativity scale using the linear relationship $\chi=A \times\left(100-2 p_{z}(\%)\right)+B$. By matching the upper and lower physical limits of the new scale to Pauling's, i.e., $\chi(66.66 \%)=0.7$ for atomic Fr and $\chi(38.1 \%)=4$ for atomic $\mathrm{F}$, we find $A=0.1155$ and $B$ $=-3.152$. We estimate our calculated values of $2 p_{z}(\%)$ to be uncertain by at most $\pm 3 \%$, as can be seen by comparison of experiment and theory (e.g., for $\mathrm{HCl}$ and $\mathrm{CF}_{3} \mathrm{Cl}$, the experimental $2 p_{z}$ populations are $54 \%$ and $52 \%$, respectively, while they are $52 \%$ and $51 \%$ from theory; see also Ref. 44, where $2 p_{z}=52 \%$ for $\mathrm{HCl}$ ). As a result, electronegativities

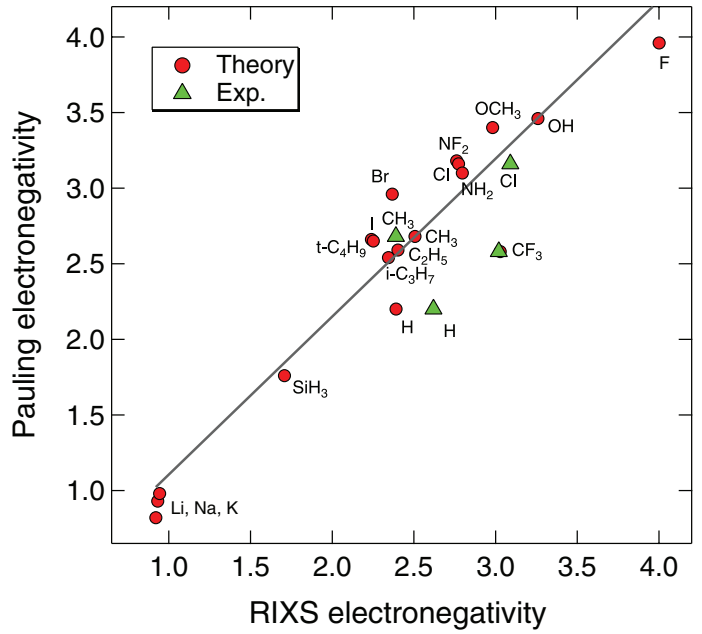

FIG. 7. Pauling electronegativities versus present RIXS electronegativities. The linear fit gives $\chi($ Pauling $)=1.0446 \times \chi($ RIXS $)+0.061$.

determined with the new scale could be uncertain by as much as \pm 0.4 . Nevertheless, this approach provides a new approach to electronegativity based on charge localization in a LUMO upon core-electron excitation, a parameter that is directly related to the polarity of the Cl-R chemical bond. This last point is consistent with one of the main concepts of the Pauling scale, to estimate, using bond-dissociation energies, the polarity of chemical bonds and the net charge on each atom (see Eq. (1)).

\section{B. From the free radical to the molecule: Geometry dependencies of absolute electronegativity/hardness}

With a new electronegativity scale in hand, it is relevant to compare it to existing scales based on other approaches. For atomic ligands, the derived electronegativities correlate well with the Pauling/Datta and XPS scales, i.e., $\chi(\mathrm{F})>\chi(\mathrm{Cl})$ $>\chi(\mathrm{Br})>\chi(\mathrm{I})>\chi(\mathrm{Li})>\chi(\mathrm{Na})>\chi(\mathrm{K})$.

For alkyl and similar ligands $\left(\mathrm{C}_{p} \mathrm{H}_{2 p+1}, \mathrm{p}=1-4\right)$ and $\mathrm{C}(\mathrm{H}, \mathrm{F})_{3}$, our calculated electronegativities yield the following ordering: $\chi\left(\mathrm{C}\left(\mathrm{CH}_{3}\right)_{3}\right)<\chi\left(\mathrm{i}-\mathrm{C}_{3} \mathrm{H}_{7}\right)$ $<\chi\left(\mathrm{C}_{2} \mathrm{H}_{5}\right)<\chi\left(\mathrm{CH}_{3}\right)<\chi\left(\mathrm{CF}_{3}\right)$, adhering to the common notion that alkyl groups, which are electron-releasing, become more so with increasing size. This order differs from the Pauling/Datta scale, where $\chi\left(\mathrm{C}\left(\mathrm{CH}_{3}\right)_{3}\right)$ is larger than $\chi\left(\mathrm{i}-\mathrm{C}_{3} \mathrm{H}_{7}\right)$ and $\chi\left(\mathrm{C}_{2} \mathrm{H}_{5}\right)$ due to a spurious steric effect. ${ }^{11}$

For other ligands, the broad features generally match those of other methods (e.g., $\chi\left(\mathrm{CH}_{3}\right)<\chi\left(\mathrm{CF}_{3}\right)$, and $\chi\left(\mathrm{SiH}_{3}\right)<\chi\left(\mathrm{SiCl}_{3}\right)<\chi\left(\mathrm{SiF}_{3}\right)$, as expected $)$. However, there are a few exceptions, namely $\mathrm{C}\left(\mathrm{CH}_{3}\right)_{3}, \mathrm{SiH}_{3}, \mathrm{H}, \mathrm{CH}_{3}$, and $\mathrm{CF}_{3}$, for which the current approach has the electronegativities increasing in the following order: $\chi\left(\mathrm{SiH}_{3}\right)(1.70)$ $<\chi\left(\mathrm{C}\left(\mathrm{CH}_{3}\right)_{3}(2.25)<\chi\left(\mathrm{CH}_{3}\right)_{\exp }(2.39)<\chi(\mathrm{H})_{\exp }(2.62)\right.$ $<\chi\left(\mathrm{CF}_{3}\right)_{\text {exp }}$ (3.02), differing somewhat from other approaches such as XPS, Pauling/Datta, and Pearson. 5,45

The Pearson scale suffers for groups in which the geometry of the isolated species differs significantly from the geometry of the same species bound in a molecule. For both the $\mathrm{SiH}_{3}$. radical and the $\mathrm{SiH}_{3} \mathrm{Cl}$ molecule, the silyl group has 
TABLE II. Calculated IP, EA, electronegativity, and hardness ( $\eta$ ), in $\mathrm{eV}$, at the equilibrium geometries of the $\mathrm{t}_{-} \mathrm{C}_{4} \mathrm{H}_{9}, \mathrm{SiH}_{3}$, and $\mathrm{CH}_{3}$ free radicals, compared to their frozen geometries in $\mathrm{t}-\mathrm{C}_{4} \mathrm{H}_{9} \mathrm{Cl}, \mathrm{SiH}_{3} \mathrm{Cl}$, and $\mathrm{CH}_{3} \mathrm{Cl}$ molecules. Charges on the chlorine centers $(\mathrm{Q})$ are calculated using Eq. (14), $\chi$, and $\eta$ of $\mathrm{Cl}(8.31 \mathrm{eV}, 4.70 \mathrm{eV})$. The Coulombic interaction $\left(\mathrm{J}_{\mathrm{Cl}-G}\right)$ is calculated by considering the distance between chlorine and the center of mass of the group G. Experimental values are shown in parentheses. ${ }^{5}$

\begin{tabular}{|c|c|c|c|c|c|c|}
\hline & \multicolumn{2}{|r|}{$\mathrm{t}-\mathrm{C}_{4} H_{9}$} & \multicolumn{2}{|c|}{$\mathrm{SiH}_{3}$} & \multicolumn{2}{|c|}{$\mathrm{CH}_{3}$} \\
\hline & Frozen & Optimized & Frozen & Optimized & Frozen & Optimized \\
\hline IP & 7.91 & $6.97(6.93)$ & 8.86 & $8.81(8.14)$ & 10.96 & 9.78 (9.82) \\
\hline EA & 0.03 & $-0.42(-0.30)$ & 0.89 & $0.87(1.41)$ & 0.34 & $0.05(0.08)$ \\
\hline$\chi$ & 3.97 & $3.28(3.31)$ & 4.87 & $4.84(4.78)$ & 5.65 & $4.92(4.96)$ \\
\hline$\eta$ & 3.94 & $3.70(3.61)$ & 3.98 & $3.97(3.37)$ & 5.35 & $4.87(4.87)$ \\
\hline $\mathrm{Q}_{C l}$ & -0.92 & & -0.83 & & & -0.52 \\
\hline
\end{tabular}

an umbrella geometry, and the DFT/B3LYP calculated values for $\chi_{\text {radical }}(4.84 \mathrm{eV})$ and $\chi\left(\mathrm{SiH}_{3} \mathrm{Cl}\right)(4.87 \mathrm{eV})$ are found to be similar to the experimental value $(4.78 \mathrm{eV})^{5}$ (see Table II). In contrast, while the free $\mathrm{CH}_{3}$ radical is planar, the methyl group adopts an umbrella conformation in $\mathrm{CH}_{3} \mathrm{Cl}$ and many other molecules.

As expected, for the planar configuration of $\mathrm{CH}_{3}$, the calculated value $\left(\chi_{\text {radical }}=4.92 \mathrm{eV}\right)$ is very close to the experimental value $(4.96 \mathrm{eV}),{ }^{5}$ and nearly equal to the silyl group electronegativity, a general trend reproduced well by DFT calculations with different exchange-correlation functionals (see Refs. 5 and 47). But for an umbrella-shaped methyl group, the electronegativity is significantly larger $(5.65 \mathrm{eV})$ than for the free radical.

To illustrate the significance of geometrical effects, we plot in Fig. 8 electronegativity values for $\mathrm{CH}_{3}$ determined from a linear fit between $\chi$ (Pauling) and $\chi$ (Pearson) for several atomic species. This plot shows the magnitude of the change in $\chi\left(\mathrm{CH}_{3}\right)$ in its different geometries, based on calculated values of $\chi$ (Pearson) from Table II. As expected, a significant change in $\chi\left(\mathrm{CH}_{3}\right)$ is seen in going from the planar geometry (1.77) to the umbrella geometry (2.04), the latter approaching the RIXS (2.39) and Pauling (2.68) values.

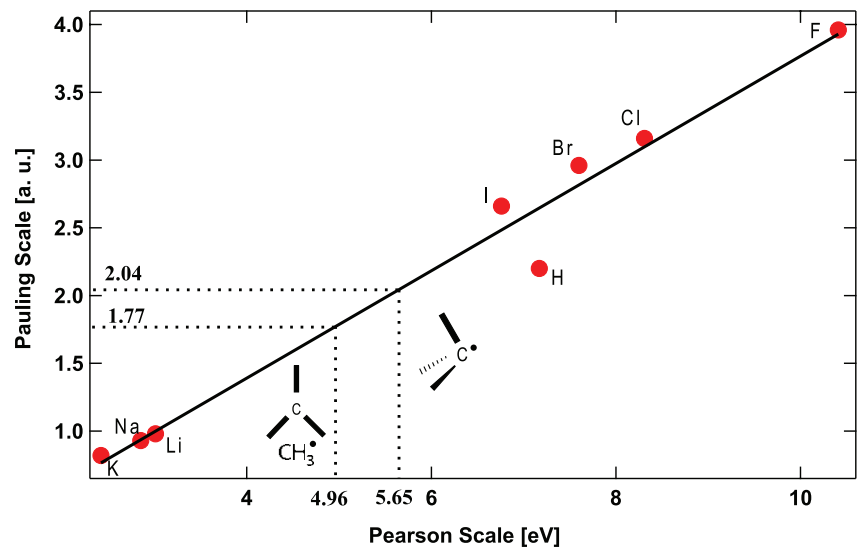

FIG. 8. Pauling electronegativities versus Pearson electronegativities for atoms. Calculated electronegativities of $\mathrm{CH}_{3}$ for planar (free radical) configuration and umbrella geometry in $\mathrm{CH}_{3} \mathrm{Cl}$. Linear fit between $\chi$ (Pauling) against $\chi$ (Pearson) for several atomic species have been considered.
A similar effect is found for $\mathrm{t}^{-} \mathrm{C}_{4} \mathrm{H}_{9}$, which undergoes a modest change in geometry in going from a radical to being bound in a molecule; $\chi$ increases from $3.28 \mathrm{eV}$ in the equilibrium geometry to $3.97 \mathrm{eV}$ for the frozen geometry, lower than $\chi\left(\mathrm{SiH}_{3}\right)$ in either geometry. The fit in Fig. 8 suggests $\chi$ to be $\approx 1.40$ for $\mathrm{t}^{-\mathrm{C}_{4} H_{9}}$ and $\approx 1.70$ for $\mathrm{SiH}_{3}$ on the Pauling scale. However, the values of $\chi$ (Pauling) extrapolated from Fig. 8 for $\mathrm{t}-\mathrm{C}_{4} \mathrm{H}_{9}, \mathrm{SiH}_{3}$, and $\mathrm{CH}_{3}$ exhibit a different ordering than seen with either the $\chi$ (Pauling) or $\chi$ (RIXS) scales (see Table I). As discussed in Sec. II A, an explanation for this difference is that any electronegativity scale derived from experimental measurements yields effective values of $\chi$ because probing the environment of a molecule necessarily includes at least some influence from parameters ((i) Coulomb interaction term, $\mathrm{J}_{R-C l}$ (see Eq. (5)), which depends on the specific topological nuclear framework and the electron-charge sharing in the molecule; (ii) electronegativity/hardness changes of the chlorine upon core-excitation process; (iii) repolarisation of the surrounding medium in which the atom probed $(\mathrm{Cl}$ in this case) is embedded) other than the absolute electronegativities of the bonding partners.

\section{Comparison to XPS-derived electronegativities}

It is instructive to compare the present RIXS-based approach to XPS. A comparison of RIXS- and XPS-derived electronegativities for several species (see Table I) generally shows similar trends for both scales, but some notable exceptions are discussed below. If we look at the correlation between the electronegativities and the chlorine ionization energies $^{48}$ for $\mathrm{RCl}\left(\mathrm{R}=\mathrm{t}-\mathrm{C}_{4} \mathrm{H}_{9}, \mathrm{i}-\mathrm{C}_{3} \mathrm{H}_{7}, \mathrm{C}_{2} \mathrm{H}_{5}, \mathrm{CH}_{3}, \mathrm{Cl}, \mathrm{CF}_{3}\right)$ we find an excellent correlation with $R^{2}=0.998$. There is a very close relationship between the new electronegativity scale and core-ionization energies, the ionization energies being much better correlated with this new scale than they do with the Pauling electronegativities listed in Table I of the present paper.

However, for the XPS scale defined by True et al. ${ }^{33}$ with $\mathrm{I}, \mathrm{Cl}, \mathrm{F}, \mathrm{SF}_{5}$, and $\mathrm{CF}_{3}$ considered as references, $\chi(\mathrm{H})$ is close to 3 , far from the value of 2.20 predicted by the Datta/Pauling ${ }^{11}$ and Mullay ${ }^{12}$ scales, or the bond-polarity index. ${ }^{48}$

The Mulliken-like definition of electronegativity for hydrogen yields $\chi($ Pearson $)=\frac{I_{p}+E A}{2}=7.17 \mathrm{eV}$, also inconsistent with Paulings and other empirical values for $\chi(\mathrm{H})$. The peculiar behavior of $\mathrm{H}$ follows from its unique chemistry, which arises due to its small size and lack of core electrons and because the EA for $\mathrm{H}$ is small due to the inability of the $\mathrm{H}$ orbital involved in a bond to expand as much as it does in free $\mathrm{H}^{-} .{ }^{17}$ According to Bergmann and Hinze, ${ }^{46}$ True et al. ${ }^{33}$ described three types of ligands: (1) hydrogen, with low electronegativity but high chemical hardness $(6.4 \mathrm{eV} / \mathrm{e})$, as defined by Parr and Pearson; ${ }^{4,5}$ (2) the halogens, which have a range of electronegativities and chemical hardness $(4.6<\eta$ $<8.7$ ), with the values roughly correlated to one another; and (3) a variety of other functional groups, including $\mathrm{CF}_{3}$ and $\mathrm{SF}_{5}$, with chemical hardness between 2 and 3 , uncorrelated to electronegativity. The high chemical hardness of $\mathrm{H}$ reflects its 
TABLE III. Calculated net charges on $\mathrm{Cl}$ using the Rappe-Goddard (RG) charge-equilibration model, ${ }^{17}$ compared with present HF/DFT-B3LYP/Mulliken charges for $\mathrm{t}-\mathrm{C}^{(1)}-\left(\mathrm{C}^{(2)} \mathrm{H}_{3}\right)_{3} \mathrm{Cl}, \mathrm{SiH}_{3} \mathrm{Cl}$, and $\mathrm{CH}_{3} \mathrm{Cl}$.

\begin{tabular}{lccc}
\hline \hline & $\mathrm{t}-\mathrm{C}^{(1)}\left(\mathrm{C}^{(2)} \mathrm{H}_{3}\right)_{3}$ & $\mathrm{SiH}$ & $\mathrm{CH}$ \\
& $\mathrm{Cl} / \mathrm{C}^{(1)} / \mathrm{C}^{(2)} / \mathrm{H}$ & $\mathrm{Cl} / \mathrm{Si} / \mathrm{H}$ & $\mathrm{Cl} / \mathrm{C} / \mathrm{H}$ \\
\hline $\mathrm{RG}$ & & & \\
$\mathrm{Q}_{R G}$ & $-0.56 / \approx 0.0 /-0.21 / 0.085$ & $-0.63 / 0.65 / \approx 0.0$ & $-0.45 /-0.07 / 0.17$ \\
$-\epsilon_{2 p}^{A t}+\mathrm{k}_{2 p} \mathrm{q}^{\mathrm{a}}$ & 213.10 & 212.25 & 214.40 \\
$\mathrm{~V}_{R}{ }^{\mathrm{a}}$ & 4.60 & 6.06 & 3.72 \\
DFT/Mulliken & & & $-0.25 /-0.18 / 0.14$ \\
$\mathrm{Q}_{\text {Mull. }}$ & $-0.26 /-0.16 /-0.18 / 0.11$ & $-0.30 / 0.71 /-0.14$ & 216.76 \\
$-\epsilon_{2 p}^{A t}+\mathrm{k}_{2 p} \mathrm{q}^{\mathrm{a}}$ & 216.62 & 215.96 & 1.36 \\
$\mathrm{~V}_{R}{ }^{\mathrm{a}}$ & 1.08 & 2.35 & $-0.33 /-0.08 / 0.14$ \\
$\mathrm{HF} /$ Mulliken & & & 215.84 \\
$\mathrm{Q}_{\text {Mull. }}$ & $-0.45 /-0.28 /-0.06 / 0.10$ & $-0.39 / 0.90 /-0.17$ & 2.28 \\
$-\epsilon_{2 p}^{A t}+\mathrm{k}_{2 p} \mathrm{q}^{\mathrm{a}}$ & 214.42 & 215.11 & \\
$\mathrm{~V}_{R}{ }^{\mathrm{a}}$ & 3.28 & 3.20 & \\
\hline \hline & & &
\end{tabular}

low polarizability and hence low relaxation energy. For $\mathrm{H}$, our theoretical electronegativity is found to be $\chi=2.39$, and it is 2.62 from experiment, ${ }^{40}$ in good agreement with XPS value.

An other interesting comparison to XPS can be made by considering the $\mathrm{Cl} 2 p$ core binding energies in $\mathrm{SiH}_{3} \mathrm{Cl}$ (206.22 eV), $\mathrm{CH}_{3} \mathrm{Cl}(206.24 \mathrm{eV})$, and $\mathrm{t}-\mathrm{C}_{4} \mathrm{H}_{9} \mathrm{Cl}(205.38 \mathrm{eV})$ (see Table III). The first two species have nearly identical $\mathrm{Cl}$ $2 p$ binding energies (and thus XPS-derived electronegativities) $(\Delta \mathrm{BE}=0.02 \mathrm{eV})$ that are $\approx 1 \mathrm{eV}(\Delta \mathrm{BE}=+0.88 \mathrm{eV})$ higher than that for $\mathrm{t}-\mathrm{C}_{4} \mathrm{H}_{9} \mathrm{Cl}$, suggesting similar electronegativity for $\mathrm{SiH}_{3}$ an $\mathrm{CH}_{3}$ and a lower electronegativity value for $\mathrm{t}-\mathrm{C}_{4} \mathrm{H}_{9}$. This clearly differs from the ordering found in the RIXS and Pauling scales, where $\chi\left(\mathrm{t}-\mathrm{C}_{4} \mathrm{H}_{9} \mathrm{Cl}\right)$ lies between $\chi\left(\mathrm{SiH}_{3}\right)$ and $\chi\left(\mathrm{CH}_{3}\right)$. A question thus occurs, e.g., why the XPS scale predicts similar core binding energies for both these compounds, while the $2 p_{z}$ populations are quite different in the corresponding core-excitated states? The reason of this discrepancy between RIXS and XPS is not straightforward since both techniques measure different observables. RIXS derived electronegativity scale gives information about the degree of polarization of the spectator electron depending the nature of the radical. XPS measures the core binding energy which is the energy difference between the final coreionized state and the initial neutral ground state. In order to better understand the correlation between our electronegativity scale and $\mathrm{Cl} 2 p$ core-binding energies in $\mathrm{SiH}_{3} \mathrm{Cl}, \mathrm{CH}_{3} \mathrm{Cl}$, we have used the simple theoretical approach developed by Aitken and co-workers. ${ }^{49}$

In this model, the $\mathrm{Cl} 2 p$ core-binding energy, $\mathrm{BE}\left(\mathrm{Cl}_{2 p}\right)$, is given by the expression

$$
\mathrm{BE}\left(\mathrm{Cl}_{2 p}\right)=-\epsilon_{2 p}^{A t}+\mathrm{k}_{2 p} \mathrm{q}+\mathrm{V}_{R}+\Delta \mathrm{R}+\Delta_{c o r r},
$$

where $-\epsilon_{2 p}^{A t}$ is the binding energy of a $\mathrm{Cl} 2 p$ electron in the neutral atomic chlorine, $\mathrm{k}_{2 p}$ is a constant taking into account for any partial charge on the chlorine site if the atom experiences a partial valence electronic charge of $\mathrm{q}, \mathrm{V}_{R}$ is the polarized molecular medium producing a potential $\mathrm{V}_{R} / \mathrm{e}$ at the chlorine site, yielding an energy of $\mathrm{qV}_{R}, \Delta \mathrm{R}$ is the relaxation energy upon core ionization and $\Delta_{\text {corr }}$ is the differential cor- relation energy between the core-ionized state and the neutral gound state.

In principle, both these latter quantities have to be considered to correctly describe the final state. However, as seen in Table IV, the relaxation energy $\left(\triangle S C F-\epsilon_{2 p}^{K T}\right)$ is nearly the same $(\approx 12 \mathrm{eV})$ for $\mathrm{CH}_{3} \mathrm{Cl}$ and $\mathrm{SiH}_{3} \mathrm{Cl}$. Furthermore, the calculated energy differences between the theoretical DFT/B3LYP- $\triangle K S$ and $\triangle S C F$ core binding energies indicate that the differential correlation energy, $\Delta_{c o r r}$, is of the same order of magnitude for both these compounds. Thus, it can be assumed that relative values of $\mathrm{Cl} 2 p$ binding energies in $\mathrm{CH}_{3} \mathrm{Cl}, \mathrm{SiH}_{3} \mathrm{Cl}$ are mainly derived by initial-state (chemical shift) effects, and $\Delta \mathrm{BE}\left(\mathrm{Cl}_{2 p}\right)$ can be reduced in the present case, to

$$
\Delta \mathrm{BE}\left(\mathrm{Cl}_{2 p}\right) \approx \Delta \mathrm{V},
$$

where $\mathrm{V}=\mathrm{k}_{2 p} \mathrm{q}+\mathrm{V}_{R}$ reflects not only the charge on the chlorine but also on the local electrostatic potential created by

TABLE IV. Calculated $-\epsilon_{2 p}{ }^{K T}$ (in eV) within the Frozen-core approximation (Koopmans Theorem (KT)) as derived from HF ground-state calculations. Calculated $\triangle \mathrm{SCF}$ core-binding energy (in $\mathrm{eV}$ ). Calculated $\Delta \mathrm{V}$ and $\Delta \mathrm{R}$ (see text, in eV). ${ }^{\mathrm{a}} \mathrm{CH}_{3} \mathrm{Cl}$ is chosen as reference. Calculated Kohn-sham $(\triangle \mathrm{KS})$ core binding energies with $\mathrm{B} 3 \mathrm{LY} \mathrm{P}^{50,51}$ exchange-correlation functional. $\Delta_{\text {corr }}=\mathrm{B} 3 \mathrm{LYP} / \triangle K S-\triangle S C F($ in $\mathrm{eV})$. Measured $\mathrm{Cl} 2 \mathrm{p}_{3 / 2}$ core binding energies from Ref. 26. DFT/B3LYP valence-shell electronic transfer (CT) from the ligand to the core-ionized(excited) chlorine. Calculated LUMO (Mulliken) electronic population of chlorine $\left(\operatorname{Pop}_{C l *}^{L U O}\right)$ in the core-excited state.

\begin{tabular}{lccc}
\hline \hline & $\mathrm{t}-\mathrm{C}\left(\mathrm{CH}_{3}\right)_{3}$ & $\mathrm{SiH}_{3} \mathrm{Cl}$ & $\mathrm{CH}_{3} \mathrm{Cl}$ \\
\hline$-\epsilon_{2 p}{ }^{K T}$ & 217.70 & 218.31 & 218.12 \\
$\Delta \mathrm{V}$ & $-0.42\left(-0.25^{a}\right)$ & 0.19 & 0.0 \\
$\Delta \mathrm{SCF}$ & 205.24 & 206.29 & 206.21 \\
$\Delta \mathrm{R}$ & $0.55\left(0.63^{a}\right)$ & 0.11 & 0.0 \\
$\Delta \mathrm{KS}\left(\Delta_{\text {corr }}\right)$ & $205.40(0.16)$ & $206.33(0.04)$ & $206.43(0.22)$ \\
$\mathrm{Cl} 2 p_{3 / 2}^{-1}($ Expt, in eV) & 205.38 & 206.22 & 206.24 \\
$\mathrm{CT}_{\text {val. }}^{\text {ion }}$ & $0.42 \mathrm{e}^{-}$ & $0.35 \mathrm{e}^{-}$ & $0.33 \mathrm{e}^{-}$ \\
$\mathrm{CT}_{\text {val }}^{\text {ex }}$ & $0.38 \mathrm{e}^{-}$ & $0.35 \mathrm{e}^{-}$ & $0.35 \mathrm{e}^{-}$ \\
Pop $_{\text {Cl* }}^{L U M O}$ & $0.33 \mathrm{e}^{-}$ & $\approx 0.0$ & $0.37 \mathrm{e}^{-}$ \\
\hline \hline
\end{tabular}

${ }^{\text {a}}$ Experimental values from Ref. 48. 
the surrounding medium. Both these quantities can compete. We have numerically estimated the potentials $\mathrm{V}_{R}$ for both the molecular systems of interest. The calculated values of the potentials $\mathrm{V}_{R}$ produced by radical onto chlorine center, are easily calculated from the expression

$$
\mathrm{V}^{R}=-\epsilon_{2 p}^{K T}+\epsilon_{2 p}^{A t}-\mathrm{k}_{2 p} \mathrm{q} .
$$

From Hartree-Fock calculations of atomic $-\epsilon_{C l_{2 p}}$ for $\mathrm{q}=-1$, 0 , and 1 , we have estimated $\mathrm{k}_{2 p} \approx 11.7 \mathrm{eV} / \mathrm{e}$, and $\epsilon_{2 p}^{A t}$ $=219.65 \mathrm{eV}$. In order to evaluate the charge of chlorine in $\mathrm{SiH}_{3} \mathrm{Cl}$ and $\mathrm{CH}_{3} \mathrm{Cl}$, we use the popular charge-equilibration model developed by Rappe and Goddard, ${ }^{17}$ and compare them to HF/DFT-B3LYP Mulliken charges calculated in this study.

It is important to note that net charge arises from two counterbalancing effects. On one hand, the $\mathrm{Cl}$ atom competes with the rest of the molecule for electron density, an effect driven by the difference in electronegativity. On the other hand, the sum of the hardness $(\eta)$ and the Coulomb potential on the $\mathrm{Cl}$ atom acts to resist any charge transfer. Using calculated values ${ }^{5}$ of $\chi$ and $\eta$ for $\mathrm{SiH}_{3}(\chi=4.87 \mathrm{eV}, \eta$ $=3.98 \mathrm{eV})$ and $\mathrm{CH}_{3}(\chi=5.65 \mathrm{eV}, \eta=5.3 \mathrm{eV}$ in the frozen umbrella geometry), and calculating $\mathrm{J}_{\mathrm{Cl}-\mathrm{G}}$ using the distance between chlorine and the center of mass of the group $\mathrm{G}$, we estimate the net charge on the $\mathrm{Cl}$ atom in each molecule as

$$
\mathrm{Q}_{\mathrm{Cl}}=\frac{\left(X_{G}^{o}-X_{C l}^{o}\right)}{2\left(\eta_{C l}+\eta_{G}-J_{C l-G}\right)} .
$$

We find $\mathrm{Q}_{\mathrm{SiH}_{3}}=-0.83$ and $\mathrm{Q}_{\mathrm{CH}_{3}}=-0.52$. Examining the net charges calculated for these two molecules using three other different approaches (see Table III), in spite we find large variations depending on the model used, the $\mathrm{Cl}$ in $\mathrm{SiH}_{3} \mathrm{Cl}$ is consistently found to have a lower net charge than the $\mathrm{Cl}$ in methyl chloride. However, the polarizable radicals produce different potentials at the chlorine site, yielding systematically for $\mathrm{SiH}_{3}$ a larger $\mathrm{V}_{R}$, the positive charge on $\mathrm{CH}_{3}$ being localized mainly on the $\mathrm{H}$ atoms and thus is less effective at producing a positive potential at the $\mathrm{Cl}$ atom than is the positive charge on the $\mathrm{Si}$ atom in $\mathrm{SiH}_{3}$. Furthermore, as reported in Table IV, Mulliken population analysis shows that the amount of charge transfer that occurs through re-polarization of the valence-shell electrons in the core-ionized state is comparable for $\mathrm{CH}_{3} \mathrm{Cl}$ and $\mathrm{SiH}_{3} \mathrm{Cl}$. Thus, our interpretation is that, in spite of different charge on chlorine due to different electronegativity of $\mathrm{SiH}_{3}$ and $\mathrm{CH}_{3}$ (see Pauling scale), the effect of polarizable radicals producing different potentials, $\mathrm{V}_{R}$, at the chlorine site, combined with a same amount of electron transfer due to electronic relaxation effects after core-ionization process, yields similar experimental core binding energies.

As reported in Table IV, Mulliken population analysis shows that the amount of charge transfer that occurs through re-polarization of the valence-shell electrons in the coreionized state is comparable to that in the core-excited state of $\mathrm{CH}_{3} \mathrm{Cl}$ and $\mathrm{SiH}_{3} \mathrm{Cl}$. Nevertheless, the spectator electron in the LUMO actually has more density on the core-excited chlorine in $\mathrm{CH}_{3} \mathrm{Cl}\left(0.37 \mathrm{e}^{-}\right)$than it has on the chlorine in $\mathrm{SiH}_{3}(\approx 0.0)$. This indicates that the LUMO charge-sharing magnitude between $\mathrm{Cl}$ and the surrounding ligands, e.g., $\mathrm{SiH}_{3} \mathrm{vs}^{\mathrm{CH}_{3}}$, still bears the memory of the electronegativity difference between these radicals, as predicted by the Pauling scale.
As shown in Table $\mathrm{IV}$, the calculated $\mathrm{Cl} 2 p$ relaxation free $-\epsilon_{2 p}{ }^{K T}$ and $\Delta \mathrm{SCF}$ binding energies for $\mathrm{t}-\mathrm{C}_{4} \mathrm{H}_{9} \mathrm{Cl}$ are the lowest in both cases, in agreement with experiment. This result can be explained by the addition of two different contributions, no matter the value of the net charge for $\mathrm{t}-\mathrm{C}_{4} \mathrm{H}_{9} \mathrm{Cl}$ relative to those of $\mathrm{SiH}_{3} \mathrm{Cl}$ and $\mathrm{CH}_{3} \mathrm{Cl}$ and using the different (RG/DFT/HF) approaches, (i) the potential at the $\mathrm{Cl}$ atom due to the $\mathrm{t}_{-} \mathrm{C}_{4} \mathrm{H}_{9}$ group, at which the positive sign of $\mathrm{V}_{R}$ is due to the positive net charge on the hydrogen atoms of the tertiary methyl groups located far from the chlorine atom and (ii) due to its larger size and higher polarizability, the relaxation energy for $\mathrm{t}-\mathrm{C}_{4} \mathrm{H}_{9} \mathrm{Cl}$ being slightly larger $(\approx 12.5 \mathrm{eV})$ compared to $\mathrm{SiH}_{3} \mathrm{Cl}$ and $\mathrm{CH}_{3} \mathrm{Cl}$.

\section{COMPUTATIONAL METHODS AND EXPERIMENT}

\section{A. Resonant inelastic x-ray scattering: Theoretical model}

The spectral and polarization properties of RIXS are derived from the scattering cross section which is based on the Kramers-Heisenberg formula:

$\sigma_{o}\left(\omega_{1}, \omega_{2}\right)=r_{o}^{2} \frac{\omega_{2}}{\omega_{1}} \sum_{f}\left|F_{f}\left(\omega_{1}, \vec{e}_{1}, \vec{e}_{2}\right)\right|^{2} \delta\left(\omega_{2}+\omega_{f o}-\omega_{1}\right)$,

where $\mathrm{F}_{f}\left(\omega_{1}, \vec{e}_{1}, \vec{e}_{2}\right)$ is the stationary scattering amplitude,

$$
F_{f}=\sum_{c} \frac{\left\langle f\left|\vec{D} \cdot \vec{e}_{2}\right| c\right\rangle\left\langle c\left|\vec{D} \cdot \vec{e}_{1}\right| o\right\rangle}{\left(\omega_{1}-\omega_{c o}\right)+\imath \Gamma_{c} / 2},
$$

$\omega_{c o}$ corresponds to the transition energy from the ground state $(|o\rangle)$ to the $1 s^{-1} L U M O^{1}$ intermediate core excited state $(|c\rangle)$, $\omega_{1}, \vec{e}_{1}$ and $\omega_{2}, \vec{e}_{2}$ are the frequencies and polarization vectors of the incident and scattered photons, respectively, $\Gamma_{c}$ is the lifetime broadening (FWHM) of the intermediate state $|c\rangle$. In a real experiment, the RXS spectral shape is distorted due to Finite width of the spectral function of the incident $\mathrm{x}$-ray radiation, where $\Phi\left(\omega_{1}-\omega_{o}, \gamma_{p}\right)$ is the spectral function of the incident light, centered at $\omega_{o}$ frequency with $\gamma_{p}$ of photon bandwidth and the spectrometer resolution, $\Phi^{\prime}\left(\omega_{2}-\omega^{\prime}, \gamma_{s}\right)$. To account for these effects, we need to convolute the spectrum with the respective spectral functions.

$$
\begin{aligned}
\sigma\left(\omega_{o}, \omega^{\prime}\right)= & \iint d \omega_{1} d \omega_{2} \sigma_{o}\left(\omega_{1}, \omega_{2}\right) \Phi\left(\omega_{1}-\omega_{o}, \gamma_{p}\right) \\
& \times \Phi^{\prime}\left(\omega_{2}-\omega^{\prime}, \gamma_{s}\right)
\end{aligned}
$$

Due to energy conservation law, the above expression can be rewritten as

$$
\begin{aligned}
\sigma\left(\omega_{o}, \omega^{\prime}\right)= & r_{o}^{2} \iint d \omega_{1} d \omega_{2} \frac{\omega_{2}}{\omega_{1}} \sum_{f}\left|F_{f}\left(\omega_{2}+\omega_{f o}, \vec{e}_{1}, \vec{e}_{2}\right)\right|^{2} \\
& \times \Phi\left(\omega_{2}+\omega_{f o}-\omega_{o}, \gamma_{p}\right) \Phi^{\prime}\left(\omega_{2}-\omega^{\prime}, \gamma_{s}\right),
\end{aligned}
$$

where the corresponding scattering amplitude in RIXS is given now by the expression

$$
F_{f}=\sum_{c} \frac{\left\langle f\left|\vec{D} \cdot \vec{e}_{2}\right| c\right\rangle\left\langle c\left|\vec{D} \cdot \vec{e}_{1}\right| o\right\rangle}{\left(\omega_{2}-\omega_{c f}\right)+\imath \Gamma_{c} / 2},
$$


where $\hbar \omega_{c f}$ is the transition energy between the intermediate state, $|c\rangle$, and the final state, $|f\rangle$.

\section{B. Spin-orbit coupling}

In all the molecules (Cl-R) considered here, the LUMO orbital has antibonding character, and its electron density is localized along the Cl-R bond and shared to varying degrees between the chlorine atom and the ligand R. The final $\mathrm{Cl} 2 p^{-1} L U M O^{1}$ relativistic spin-orbit states are a mixture of ${ }^{1,3} \Pi$, and ${ }^{1,3} \Sigma$ singlet and triplet states. To model these states, the spin-orbit interaction can be written as an effective Hamiltonian,

$$
H_{s o}=\sum_{i=1}^{n_{e l}} \hat{P}_{2 p}\left(\gamma \cdot \hat{\mathbf{s}}_{\mathbf{i}} \cdot \hat{\mathbf{l}}_{\mathbf{i}}\right) \hat{P}_{2 p}
$$

where $\hat{P}_{2 p}$ is the projection operator on the chlorine $2 p$ orbitals, $\hat{\mathbf{s}}_{\mathbf{i}}$ and $\hat{\mathbf{l}}_{\mathbf{i}}$ are the spin and angular-momentum operators of the ith electron, and $\gamma$ is the spin-orbit coupling parameter for the $\mathrm{Cl} 2 p$ subshell. As shown in Ref. 43, for the $2 p^{-1} \mathrm{LUMO}^{1}$ state, a few assumptions lead to simplified spinorbit Hamiltonians in the basis of the nonrelativistic wavefunctions, ${ }^{1,3} \Pi$ and ${ }^{1,3} \Sigma$ :

$$
\begin{aligned}
& H\left(2 p^{-1} \sigma, M_{J}=0\right)=\left|\begin{array}{cc}
E\left({ }^{3} \Pi\right)+\gamma / 2 & \gamma / \sqrt{2} \\
\gamma / \sqrt{2} & E\left({ }^{1} \Sigma\right)
\end{array}\right|, \\
& H\left(2 p^{-1} \sigma, M_{J}=1\right)=\left|\begin{array}{ccc}
E\left({ }^{3} \Pi\right) & \gamma / 2 & \gamma / 2 \\
\gamma / 2 & E\left({ }^{3} \Sigma\right) & \gamma / 2 \\
\gamma / 2 & \gamma / 2 & E\left({ }^{1} \Pi\right)
\end{array}\right| .
\end{aligned}
$$

Upon diagonalization, the relativistic wavefunctions are found to be a mixture of singlet and triplet states, and, apart from any molecular-field splitting, the energies of these spinorbit states are affected by singlet-triplet splitting. In fact, due to the combination of spin-orbit coupling and molecular-field splitting, the $2 p^{-1} \mathrm{LUMO}^{1}$ configuration splits into 12 sublevels, corresponding to $J_{Z}=2,1,1,0$. However, due to selection rules, only the 8 that include singlet $2 p_{x, y, z}$ components contribute to the RIXS spectra, and, furthermore, sub-levels that include either the $2 p_{x}$ or the $2 p_{y}$ nonrelativistic states are degenerate.

For randomly oriented molecules, the mean-square scattering amplitude has to be averaged over all spatial orientations. The mean-square amplitude of diffusion is given by Refs. 52 and 53

$$
\left\langle\left|F_{2 p_{\gamma} v a l^{*}}\right|^{2}\right\rangle=A \lambda_{2 p_{\gamma} v a l^{*}}^{\alpha \alpha \beta \beta}+B \lambda_{2 p_{\gamma} v a l^{*}}^{\alpha \beta \alpha \beta}+C \lambda_{2 p_{\gamma} v a l^{*}}^{\alpha \beta \beta \alpha},
$$

where we have introduced

$$
\lambda_{2 p_{\gamma} v a l^{*}}^{\alpha \beta \beta \alpha}=\sum_{\alpha=x, y, z} \sum_{\beta=x, y, z} F_{2 p_{\gamma} v a l^{*}}^{\alpha \beta} F_{2 p_{\gamma} v a l^{*}}^{\beta \alpha^{*}}
$$

where $2 \mathrm{p}_{\gamma}$ is a spin-orbit final states and $\alpha$ and $\beta$ represent the $x, y, z$ components of the dipole operator $\mathcal{D}$. The coefficients $\mathrm{A}, \mathrm{B}$, and $\mathrm{C}$ depend only on the polarization of the incident and emitted x-rays, $\vec{e}_{1}, \vec{e}_{2}$ :

$$
\begin{aligned}
& A=-\left|\vec{e}_{1} \cdot \vec{e}_{2}^{*}\right|^{2}+4\left|\vec{e}_{1} \cdot \vec{e}_{2}^{*}\right|^{2}-1, \\
& B=-\left|\vec{e}_{1} \cdot \overrightarrow{e_{2}^{*}}\right|^{2}-\left|\overrightarrow{e_{1}} \cdot \overrightarrow{e_{2}^{*}}\right|^{2}+4, \\
& C=4\left|\vec{e}_{1} \cdot \vec{e}_{2}^{*}\right|^{2}-\left|\vec{e}_{1} \cdot \vec{e}_{2}^{*}\right|^{2}-1 .
\end{aligned}
$$

With $\theta$ as the angle between the two polarization vectors $\vec{e}_{1}$ and $\vec{e}_{2}$, the mean value of the (squared) amplitude is given by ${ }^{36,37,39}$

$$
\left\langle\left|F_{2 p_{\gamma} L U M O^{*}}\right|^{2}\right\rangle=2\left(1+2 \cos ^{2} \theta\right)\left|F_{2 p_{z} L U M O^{*}}^{z z}\right|^{2} W_{\gamma, S}^{z},
$$

for ${ }^{1} \Sigma$ states and

$$
\left\langle\left|F_{2 p_{\gamma} L U M O^{*}}\right|^{2}\right\rangle=4\left(2-\cos ^{2} \theta\right)\left|F_{2 p_{x} L U M O^{*}}^{x z}\right|^{2} W_{\gamma, S}^{x},
$$

for ${ }^{1} \Pi$ states.

$W_{\gamma, S}^{z}$ and $W_{\gamma, S}^{(x, y)}$ are the nonrelativistic singlet $2 p_{z}^{-1} L U M O^{1}$ and degenerate $2 p_{(x, y)}^{-1} L U M O^{1}$ populations in the final $2 \mathrm{p}_{\gamma}^{-1} L U M O^{1}$ spin-orbit states and $\mathrm{F}_{2 p_{(x)} L U M O^{*}}^{(x) z z}$ take the following expressions:

$$
\begin{aligned}
& F_{2 p_{(x)} L U M O^{*}}^{(x) z} \\
& =\frac{\left\langle 2 p_{(x) z}^{-1} L U M O^{*}|(x) z| 1 s^{-1} L U M O^{*}\right\rangle\left\langle 1 s^{-1} L U M O|z| O\right\rangle}{\left(\omega_{2}-\omega_{c f}\right)+\imath \Gamma_{c} / 2} .
\end{aligned}
$$

These formula include the polarization dependence of the singlet $2 p_{(x, y)}^{-1}$ and $2 p_{z}{ }^{-1}$ components, with $W_{\gamma, S}^{(x, y)}$ and $W_{\gamma, S}^{z}$ being parameters determinable from polarized-RIXS spectra (see Sec. III D). The RIXS cross-section takes thus the final form

$$
\begin{aligned}
\sigma\left(\omega_{o}, \omega^{\prime}\right) & =r_{o}^{2} \iint d \omega_{1} d \omega_{2} \frac{\omega_{2}}{\omega_{1}} \\
& \times \sum_{f}\left\langle\left|F_{2 p_{\gamma} L U M O^{*}}\right|^{2}\right\rangle e^{-\ln 2\left(\frac{\omega_{2}+\omega_{f o}-\omega_{o}}{\gamma_{p}}\right)^{2}} \Phi^{\prime}\left(\omega_{2}-\omega^{\prime}, \gamma_{s}\right) .
\end{aligned}
$$

\section{Ab initio calculations}

All calculations on neutral and core-excited molecules in this work were carried out using the GAMESS(US) package. ${ }^{54}$ Different levels of theory were used for the geometrical optimizations and the core-excited-state calculations, respectively. For each molecule, the ground-state geometry was optimized at a DFT level, with Becke three-parameter hybrid exchange ${ }^{50}$ and the Lee-Yang-Parr gradient-corrected correlation functional $^{51}$ (B3LYP). These calculations reproduced the experimental geometries nearly exactly at moderate computational time. In a few cases $\left(\mathrm{SiH}_{3}, \mathrm{CH}_{3}\right)$, DFT-level ionization potentials and electron affinities were calculated for both the radicals in their free equilibrium geometry and for the same groups bonded to chlorine.

The spin-orbit interaction, including the full BreitPauli coupling ${ }^{55-57}$ integral package, was used for calculations of the $\mathrm{Cl} 2 p^{-1} \mathrm{LUMO}^{1}$ core-excited states, and spinorbit-coupling calculations were performed on variational configuration-interaction (CI) wavefunctions, SO-CI. The CI 
active space included the three occupied $2 p_{x, y, z}$ inner shells and the 80 lowest unoccupied virtual orbitals, all derived from the nonrelativistic $2 s^{-1} v a l^{*}$ potential. The core-excited reference wavefunction was represented by a generalized $2 s^{-1} v a l^{*}$ singlet state to avoid orientational effects artificially induced in the calculations using $2 p_{x, y, z}^{-1} v a l^{*}$ states. Because the $2 s$ and $2 p$ orbitals are close in energy $(\Delta E \sim 70 \mathrm{eV})$, equivalent relaxation effects are expected.

To treat electronic relaxation of the valence orbitals in response to the core hole accurately, a large aug-cc-pCVQZ basis set was used for chlorine. For the atom(s) of the ligands bonded to chlorine, a $6-311 \mathrm{G}^{* *}$ basis set was used, augmented by a set of $(3 s, 3 p, 3 d)$ diffuse functions. The choice of different basis sets for $\mathrm{Cl}$ and $\mathrm{R}$ was made for the following reason. To obtain calculated core-excited final states in molecules, a localized description of the hole is necessary to properly represent electronic relaxation (screening) around the core hole. This problem of core-hole localization can arise when two core levels are close in energy, even if they are located on different atomic centers in the molecular system. In the case of symmetric molecules (e.g., $\mathrm{Cl}_{2}$ ), the core hole may jump from one center to the other during successive SCF iterations, preventing convergence in the calculation. Convergence of the SCF procedure can be assured by adding a charge to the ionized nucleus, which is then diminished gradually, ${ }^{58}$ either by applying effective core potentials to the equivalent centers, ${ }^{59}$ or by using a different basis set. In the present calculations, convergence problems were avoided by considering different basis sets for $\mathrm{Cl}$ and $\mathrm{R}$. In a few cases, comparison to full calculations using a larger cc-pVQZ basis set on the ligand atoms indicated the procedure described above leads to only moderate discrepancies $(\approx 1 \%)$ in the $2 p_{z}$ calculations.

For evaluation of core binding energies and electronic populations, we used the popular theoretical model proposed by Rappe and Goddard. ${ }^{17}$ This approach to predicting charge distributions in molecules is based on experimental atomic ionization potentials, electron affinities, and atomic radii, and allows construction of an atomic chemical potential using shielded electrostatic interactions among all the charges. By requiring equal chemical potentials, equilibrium electronic charges can be determined as a function of geometry. It has been shown that this charge-equilibrium approach leads to charges in excellent agreement with experimental dipole moments and with atomic charges obtained from electrostatic potentials of accurate $a b$ initio calculations. As suggested by Rappe and Goddard, ${ }^{17}$ a charge-dependent parameter was used for an accurate description of the charge on hydrogen.

\section{Instrumentation and measurement}

Polarized-RIXS measurements were performed with a Rowland-circle x-ray-emission spectrometer (see Fig. 1) on beamline 9.3.1 at the ALS and have been described previously. ${ }^{60}$ The beamline provides an intense photon beam $\sim 100 \%$ linearly polarized in the horizontal plane, focused into a gas cell containing up to $\sim 400 \mathrm{mbar}$ of a sample. The $\mathrm{x}-$ ray-scattering plane is vertical, and the scattered beam is both energy analyzed and focused onto a position sensitive detec- tor by a curved $\mathrm{Si}(111)$ crystal, yielding an energy resolution of $400 \mathrm{meV}$ near the $\mathrm{Cl} K$ edge. As the Bragg angle for $\mathrm{Cl} K L$ emission is very close to the Brewster angle for $\mathrm{x}$-rays $\left(45^{\circ}\right)$, the crystal reflectivity of the perpendicular component of the polarization approaches zero and only parallel-polarized $\mathrm{x}-$ rays are diffracted efficiently; i.e., the diffracting crystal acts as an efficient $\mathrm{x}$-ray polarimeter. By rotating the spectrometer, we are able to perform polarized-RIXS measurements as a function of the angle $\theta$ between the incident $\left(\vec{e}_{1}\right)$ and scattered $\left(\overrightarrow{e_{2}}\right)$ polarization vectors. ${ }^{36,37}$ For the experimental results reported here, $K L$ emission lines were measured after resonant photoexcitation at the $\mathrm{Cl} 1 \mathrm{~s} \rightarrow \mathrm{LUMO}$ resonance for ten different angles $\theta$. Experimental spin-orbit ratios, $\mathbf{R}$ $=\mathrm{I}\left(2 p_{3 / 2}\right) / \mathrm{I}\left(2 p_{1 / 2}\right)$, were determined for each value of $\theta$ from a least-squares fit of the two $K L$ emission lines by two Voigt profiles each, assuming identical profiles for the spin-orbit doublet, $2 p_{3 / 2}$ and $2 p_{1 / 2}$ (see Fig. 4).

The spin-orbit ratio, $\mathrm{R}$, measured experimentally as a function of $\theta$ can be related to $W_{\gamma, S}^{(x, y)}$ and $W_{\gamma, S}^{z}$ (see Sec. III B). Indeed, R can be fitted to the following equation and the populations $W_{1 / 2}^{(x, y)}$ and $W_{1 / 2}^{z}$ can be extracted directly as free parameters:

$$
\begin{aligned}
\mathrm{R} & =\frac{I\left(2 p_{3 / 2}\right)}{I\left(2 p_{1 / 2}\right)} \\
& =\frac{2\left(1+2 \cos ^{2} \theta\right)\left(1-W_{1 / 2}^{z}\right)+4\left(2-\cos ^{2} \theta\right)\left(1-W_{1 / 2}^{x}\right)}{2\left(1+2 \cos ^{2} \theta\right) W_{1 / 2}^{z}+4\left(2-\cos ^{2} \theta\right) W_{1 / 2}^{x}} .
\end{aligned}
$$

Statistical errors on the extracted values of $W_{1 / 2}^{(x, y)}$ and $W_{1 / 2}^{z}$ from this procedure suggest rather small uncertainties of about $0.5 \%$ for populations derived directly from experiment.

\section{CONCLUSION}

Electronegativity values derived from polarizationresolved resonant inelastic x-ray scattering have been reported. Supported by $a b$ initio calculations, we demonstrated that $\mathrm{Cl} K L$ emission from gas-phase molecules is extremely sensitive to coupling effects between molecular-field, singlettriplet-exchange, and spin-orbit interactions, through the variation of $\mathrm{Cl} 2 p_{z}$ populations as a function of the ligand bound to $\mathrm{Cl}$. In particular, we found a linear relationship between the nature of ligands and the $2 p z$ populations that was explained in terms of localization of the LUMO into which the core electron is excited; the net charge transfer between chlorine and its ligand, and thus the nature of the chemical bond, is probed by RIXS spectroscopy because of the delocalized nature of the LUMO. To our knowledge, this is the first time a correlation between a textbook chemical parameter and excitationdeexcitation behavior in molecules has been established.

The rearrangements of electrons that accompany the formation of a molecule are very subtle, and free-atom electronegativities may not provide a comprehensive picture of these rearrangements. The new RIXS-based approach provides an electronegativity scale highly consistent with the fundamental ideas of Pauling about the chemical bond, because 
RIXS-based values of $\chi$ are determined via direct measurements of molecular systems.

In all such experimental approaches, including RIXS and the thermochemical picture, only effective electronegativities can be determined because various factors (electronic relaxation, repolarization of the chemical bond) beyond the absolute electronegativity of an isolated species, its hardness and the potential due to the rest of the molecule are inherently present when core-excitation. Through consideration of a few cases, this work discussed how to link the XPS, Pauling, and Mulliken/Pearson approaches with the polarized-RIXS approach. We conclude that this new method, based on electrondensity analysis of the LUMOs of different substituents bound to chlorine, provides effective electronegativity values compatible in most of the cases considered, here, with the literature. Extension of this approach to the determination of electronegativities in gases, solids, and even liquids appears to be promising.

\section{ACKNOWLEDGMENTS}

The authors would like to express their gratitude to the staff of the Advanced Light Source (ALS) for their valuable help and helpful discussions with Professor A. Maquet. Support from the National Science Foundation under NSF Grant No. PHY-09-70125 is gratefully acknowledged. The ALS is supported by (U.S.) Department of Energy (DOE) (DEAC03-76SF00098). M.N.P. acknowledges the French Agence Nationale de la Recherche (ANR) for financial support in the framework of "Chair d'Excellence" program.

${ }^{1}$ L. Pauling, J. Am. Chem. Soc. 54, 3570 (1932).

${ }^{2}$ L. Pauling, Nature of the Chemical Bond (Cornell University Press, New York, 1960).

${ }^{3}$ R. S. Mulliken, J. Chem. Phys. 2, 782 (1934); 3, 573 (1935).

${ }^{4}$ R. G. Parr, R. A. Donelly, M. Levy, and W. E. Palke, J. Chem. Phys. 69, 4431 (1978)

${ }^{5}$ R. G. Pearson, Inorg. Chem. 27, 734 (1988).

${ }^{6}$ L. C. Allen, J. Am. Chem. Soc. 111, 9003 (1989).

${ }^{7}$ A. L. Allred and E. G. Rochow, J. Inorg. Nucl. Chem. 5, 264 (1958).

${ }^{8}$ R. T. Sanderson, J. Am. Chem. Soc. 105, 2259 (1983).

${ }^{9}$ W. Gordy, Phys. Rev. 69, 604 (1946); 69, 130 (1946).

${ }^{10}$ T. Chakraborty and D. C. Ghosh, Eur. Phys. J. D 59, 183 (2010).

${ }^{11}$ D. Datta and N. Singh, J. Phys. Chem. 94, 2187-2190 (1990).

${ }^{12}$ J. Mullay, J. Am. Chem. Soc. 107, 7271 (1985).

${ }^{13}$ P. Politzer, Z. P.-I. Shields, F. A. Bulat, and J. S. Murray, J. Chem. Theory Comput. 7, 377 (2011).

${ }^{14}$ L. R. Murphy, T. L. Meek, A. L. Allred, and L. C. Allen, J. Phys. Chem. A 104, 5857 (2000).

${ }^{15}$ D. C. Ghosh, J. Theor. Comput. Chem. 4, 21 (2005).

${ }^{16}$ R. P. Iczkowski and J. L. Margrave, J. Am. Chem. Soc. 83, 3547 (1961).

${ }^{17}$ A. K. Rappe and W. A. Goddard III, J. Phys. Chem. 95, 3358 (1991).

${ }^{18}$ L. J. Bellamy, The Infra-Red Spectra of Complex Molecules (Wiley, New York, 1958).

${ }^{19}$ C. J. Cobos and A. L. Capparelli, J. Fluorine Chem. 70, 155 (1995).

${ }^{20}$ C. A. Clasen and M. L. Good, Inorg. Chem. 9, 817 (1970).
${ }^{21}$ C. G. Swain and E. C. Lupton, J. Am. Chem. Soc. 90, 4328 (1968).

${ }^{22}$ C. G. Swain, S. H. Unger, N. R. Rosenquist, and M. S. Swain, J. Am. Chem. Soc. 105, 492 (1983).

${ }^{23}$ O. Travnikova, S. Svensson, D. Céolin, Z. Bao, and M. N. Piancastelli, Phys. Chem. Chem. Phys. 11, 826 (2009).

${ }^{24}$ B. Lindberg, S. Svensson, P. A. Malmquist, E. Basilier, U. Gelius, and K. Siegbahn, Chem. Phys. Lett. 40, 175 (1976).

${ }^{25}$ E. Sacher, Appl. Surf. Sci. 74, 129 (1994).

${ }^{26}$ W. L. Jolly, K. D. Bomben, and C. J. Eyerman, At. Data Nucl. Data Tables 31, 433 (1984).

${ }^{27}$ V. Myrseth, L. Sæthre, K. Børve, and T. D. Thomas, J. Org. Chem. 72, 5715 (2007).

${ }^{28}$ C. Cauletti, C. Giancaspro, A. Monaci, and M. N. Piancastelli, J. Chem. Soc. Perkin Trans. 2, 656 (1981).

${ }^{29}$ F. Gel'mukhanov et al., Phys. Rev. A 59, 380 (1999).

${ }^{30}$ F. Gel'mukhanov and H. Ågren, Phys. Rep. 312, 87 (1999).

${ }^{31}$ M. Coville and T. D. Thomas, Phys. Rev. A 43, 6053 (1991).

${ }^{32}$ T. D. Thomas, L. Sæthre, K. J. Børve, J. D. Bozek, M. Huttula, and E. Kukk, J. Phys. Chem. A 108, 4983 (2004).

${ }^{33}$ J. True, T. D. Thomas, R. W. Winter, and G. L. Gard, Inorg. Chem. 42, 4437 (2003).

${ }^{34}$ E. Kukk, J. D. Bozek, J. A. Sheehy, P. W. Langhoff, and N. Berrah, J. Phys. B 33, L51 (2000).

${ }^{35}$ S. Carniato, R. Taïeb, R. Guillemin, L. Journel, M. Simon, and F. Gel'mukhanov, Chem. Phys. Lett. 439, 402 (2007).

${ }^{36}$ R. Guillemin, S. Carniato, W. C. Stolte, L. Journel, R. Taïeb, D. W. Lindle, and M. Simon, Phys. Rev. Lett. 101, 133003 (2008).

${ }^{37}$ S. Carniato, R. Guillemin, W. C. Stolte, L. Journel, R. Taïeb, D. W. Lindle, and M. Simon, Phys. Rev. A 80, 032513 (2009).

${ }^{38}$ M. Kavčič, M. Žitnic, K. Bŭcar, A. Mihelič, S. Carniato, L. Journel, R. Guillemin, and M. Simon, Phys. Rev. Lett. 105, 113004 (2010).

${ }^{39}$ L. El Khoury, L. Journel, S. Carniato, R. Guillemin, W. C. Stolte, T. Marin, D. W. Lindle, and M. Simon, J. Chem. Phys. 136, 024319 (2012).

${ }^{40}$ R. Guillemin, W. C. Stolte, L. Journel, S. Carniato, M. N. Piancastelli, D. W. Lindle, and M. Simon, Phys. Rev. A 86, 013407 (2012).

${ }^{41}$ M. Simon, L. Journel, R. Guillemin, W. C. Stolte, I. Minkov, F. Gel'mukhanov, P. Sałek, H. Ågren, S. Carniato, R. Taïeb, A. C. Hudson, and D. W. Lindle, Phys. Rev. A 73, 020706 (2006).

${ }^{42}$ M. E. Schwartz, Chem. Phys. Lett. 5, 50 (1970).

${ }^{43}$ G. A. Sawatzky and A. Lenselink, J. Chem. Phys. 72, 3784 (1980).

${ }^{44}$ R. Fink, M. Kivilompolo, and H. Aksela, J. Chem. Phys. 111, 10034 (1999).

${ }^{45}$ R. Parr and R. G. Pearson, J. Am. Chem. Soc. 105, 7512-7516 (1983).

${ }^{46}$ R. Viva-Reyes and A. Aria, Ecletica Quim. 33, 69 (2008).

${ }^{47}$ E. J. Aitken, M. K. Bahl, K. D. Bomben, J. K. Gimzewski, G. S. Nolan, and T. D. Thomas, J. Am. Chem. Soc. 102, 4873 (1980).

${ }^{48}$ L. H. Reed and L. C. Allen, J. Phys. Chem. 96, 157 (1992).

${ }^{49}$ D. Bergman and J. Hinze, in Structure and Bonding, edited by K. D. Sen, C. K. Jorgensen (Springer-Verlag, Berlin, 1987), Vol. 66, p. 145.

${ }^{50}$ A. D. Becke, J. Chem. Phys. 98, 5648 (1993).

${ }^{51}$ C. Lee, W. Yang, and R. G. Parr, Phys. Rev. B 37, 785 (1988).

${ }^{52}$ Y. Luo, H. Ågren, and F. Gel'mukhanov, J. Phys. B 27, 4169 (1994).

${ }^{53}$ Y. Luo, H. Ågren, and F. Gel'mukhanov, Phys. Rev. A 53, 1340 (1996).

${ }^{54}$ M. W. Schmidt, K. K. Baldridge, J. A. Boatz, S. T. Elbert, M. S. Gordon, J. H. Jensen, S. Koseki, N. Matsunaga, K. A. Nguyen, S. Su, T. L. Windus, M. Dupuis, and J. A. Montgomery, Jr., J. Comput. Chem. 14, 1347 (1993).

${ }^{55}$ T. R. Furlani and H. F. King, J. Chem. Phys. 82, 5577 (1985).

${ }^{56}$ H. F. King and T. R. Furlani, J. Comput. Chem. 9, 771 (1988).

${ }^{57}$ D. G. Fedorov and M. S. Gordon, J. Chem. Phys. 112, 5611 (2000).

${ }^{58}$ S. Carniato and P. Millie, J. Chem. Phys. 116, 3521 (2002).

${ }^{59}$ B. Brena, Y. Luo, M. Nyberg, S. Carniato, K. Nilsson, Y. Alfredsson, J. Ahlund, M. Mårtensson, H. Siegbahn, and C. Puglia, Phys. Rev. B 70, 195214 (2004).

${ }^{60}$ A. C. Hudson, W. C. Stolte, D. W. Lindle, and R. Guillemin, Rev. Sci. Instrum. 78, 053101 (2007). 